\title{
Physical properties of the ionized gas and brightness distribution in NGC $4736^{\star}$
}

\author{
I. Rodrigues ${ }^{1, \star \star}$, H. Dottori ${ }^{1}$, J. Cepa ${ }^{2,3, \star \star \star}$, and J. Vílchez ${ }^{3, \star \star \star}$ \\ 1 Dep. de Astronomia, Instituto de Física, Universidade Federal do Rio Grande do Sul, CP. 15051, Porto Alegre, 91501-970, \\ RS, Brazil \\ e-mail: irapuan@if1.if.ufrgs.br; dottori@if1.if.ufrgs.br \\ 2 Departamento de Astrofísica, Facultad de Física, Universidad de La Laguna, E-38071 La Laguna, Tenerife, Spain \\ 3 Instituto de Astrofísica de Canarias, E-38200 La Laguna, Tenerife, Spain \\ e-mail: jcn@iac.es; jvm@iac.es
}

Received September 16, 1996; accepted August 27, 1997

\begin{abstract}
In this work we study the galaxy NGC 4736, using narrow band interference filters imaging centered at the emission lines $[\mathrm{OII}]_{\lambda \lambda 3727+3729}, \mathrm{H} \beta,[\mathrm{OIII}]_{\lambda 5007}$, $\mathrm{H} \alpha,[\mathrm{SII}]_{\lambda 6716+6730}$ and $[\mathrm{SIII}]_{\lambda 9070}$ and nearby continua. We have obtained sizes, positions, emission line absolute fluxes, and continua intensities for $90 \mathrm{HII}$ regions, mainly distributed in a ring-like structure of $3.2 \mathrm{kpc}$ in diameter. The $\mathrm{H} \alpha$ luminosities are in the range $37.3 \leq \log L_{\mathrm{H} \alpha} \leq$ $39.4 \mathrm{erg} \mathrm{s}^{-1}$. The HII regions size distribution presents a characteristic diameter $D_{0}=115 \mathrm{pc}$ and verifies the relation $\log \left(L_{\mathrm{H} \alpha}\right) \propto D^{3}$. The temperature of the ionizing sources and the metallicity of the HII regions are respectively in the ranges $3.410^{4} \lesssim T_{\star} \lesssim 4.010^{4} \mathrm{~K}$ and $8.5 \lesssim 12+\log (\mathrm{O} / \mathrm{H}) \lesssim 9.3$. The masses of the ionizing clusters are in the range $510^{3} \lesssim M_{\mathrm{T}} / M_{\odot} \lesssim 210^{5}$. The continua radial surface brightness distribution is better fitted by the superposition of a de Vaucouleurs', a thin and a thick exponential disk laws. The monochromatic colors show that outside the star forming ring the disk presents a younger stellar population than inside it.
\end{abstract}

Key words: galaxies: individual: NGC 4736 galaxies: abundances - galaxies: ISM - galaxies: spiral — galaxies: structure

Send offprint requests to: I. Rodrigues

* Tables 3 and 4 are only available in electronic form at the CDS via anonymous ftp to cdsarc.u-strasbg.fr (130.79.128.5) or via http://cdsweb.u-strasbg.fr/Abstract.html

$\star \star$ Brazilian CNPq fellow.

${ }^{\star \star \star}$ Visiting astronomer, Roque de los Muchachos Observatory.

\section{Introduction}

NGC 4736 (M 94) is a bright (R)Sab(r) galaxy (de Vaucouleurs 1976 - RC2), member of Canis Venatici I group (Geller \& Huchra 1983). The galaxy is well studied in its optical morphology, kinematics of the optically emitting gas (van der Kruit 1974, 1976; Buta 1984, 1988) and Hi distribution and kinematics (Bosma et al. 1977; Mulder \& van Driel 1993).

We use in this paper Mulder \& van Driel's (1993) distance to NGC 4736 (6.6 Mpc, $\left.H_{0}=50 \mathrm{~km} \mathrm{~s}^{-1} \mathrm{kpc}^{-1}\right)$. These authors give a summary of NGC 4736 basic properties.

Observations of HiI regions in external galaxies are important as tracers of high mass star formation. They provide information about the interstellar medium from which they are formed, as well as stellar population to which they give origin. Hodge \& Kennicutt (1983) have cataloged 54 HII regions of NGC 4736. They are mainly distributed in a ring of $3.2 \mathrm{kpc}$ in diameter. CCD $\mathrm{H} \alpha$ fluxes and diameters of three HiI regions were measured by Kennicutt (1988). The chemical abundances of two of them were studied by McCall et al. (1985). Kennicutt et al. (1989) studied the luminosity function of 78 HiI regions of NGC 4736, by means of photographic photometry. Oey \& Kennicutt (1993) measured the ratios [OII]/H $\beta$ and $[\mathrm{OIII}] / \mathrm{H} \beta$ of nine HII regions of NGC 4736 in an abundance study of HiI regions in early-type spiral galaxies.

In the present paper we study the physical conditions of NGC 4736 HII regions by means of CCD imaging in strong lines and nearby continua. In Sect. 2 we describe the observations and data reduction procedures. In Sect. 3 we catalogue the HII regions position, size, absolute integrated flux in the $[\mathrm{OII}]_{\lambda \lambda 3727+3729}, \mathrm{H} \beta[\mathrm{OIII}]_{\lambda 5007}, \mathrm{H} \alpha$ $[\mathrm{SII}]_{\lambda \lambda 6716+6730}$ and $[\mathrm{SIII}]_{\lambda 9070}$ lines and continua intensity at $3812 \AA, 4556 \AA, 5276 \AA, 6269 \AA$ and $9193 \AA$. We derive in Sect. 4 the HII regions physical conditions, their 
diameters distribution, luminosity function, metal abundances and ionizing sources temperature. We also discuss in Sect. 4 the spatial distribution of the nuclear emission and the possible origin of the $\mathrm{H} \alpha \mathrm{P}$-Cygni profile observed by Filippenko \& Sargent (1985) in this region.

NGC 4736 brightness distribution was discussed by different authors. Bosma et al. (1977) distinguish 5 morphological zones in this galaxy (see image in Lynds 1974 and Mulder \& van Driel 1993): A bulge $(R<480 \mathrm{pc})$; an inner spiral structure $(480 \mathrm{pc}<R<1.6 \mathrm{kpc})$ bounded by a bright inner ring where the majority of the HiI regions are located; an outer spiral structure $(1.6 \mathrm{kpc}<R<6.4 \mathrm{kpc})$, this region probably forms an oval disk; a gap of lower surface brightness; and a faint outer ring $(R \sim 10.6 \mathrm{kpc})$. Furthermore, surface photometry reveals the presence of sub-structures within Bosma's zones, such as, a $480 \mathrm{pc}$ long central bar (Kormendy 1993; Möllenhoff et al. 1995 - MMG), isophotal twisting in the region between 320 pc and 800 pc (Varela et al. 1990; Beckman et al. 1991), etc. We also discuss in this paper the morphology of NGC 4736 at different wavelengths. The high $\mathrm{S} / \mathrm{N}$ of our continua data set allows to study NGC 4736 brightness and color distributions and to infer on the stellar population radial distribution (Sect. 5). Our conclusions are given in Sect. 6 .

\section{Observations and data reductions}

The observations were made during seven consecutive nights from February, 28 to March, 06, 1989. We used a CCD camera attached to the Cassegrain $\mathrm{f} / 15$ focus of the $1.0 \mathrm{~m}$ Jacobus Kapteyn Telescope, in the Roque de los Muchachos Observatory, La Palma, Canary Islands. The detector used was a GEC CCD $400 \times 590$ pixels $(1$ pixel $=22 \mu \mathrm{m})$ and the pixel size is equivalent to $0^{\prime \prime} 303$ on the sky. Typical readout noise was 9 electons per pixel.

We performed narrow band imagery using interference filters centered on the red shifted emission lines $[\mathrm{OII}]_{\lambda \lambda 3727+3729}, \mathrm{H} \beta[\mathrm{OIII}]_{\lambda 5007}, \mathrm{H} \alpha,[\mathrm{SII}]_{\lambda 6716+6730}$ and $[\mathrm{SIII}]_{\lambda 9070}$, as well as on the continua at $3812 \AA, 4556 \AA$, $5276 \AA, 6269 \AA$ and $9193 \AA$. In Table 1 we show the main characteristics of the filters, the number of frames obtained in each filter and the total integration time per filter. Throughout the run the seeing ranged from 0.18 to 1 '. 2 , reaching exceptionally $1^{\prime \prime} 8$.

Data reductions were carried out using a Silicon Graphics INDIGO workstation, employing standard methods of the $\mathrm{IRAF}^{1}$ reduction package, removing bad pixels, cosmic ray spots, subtracting bias, normalizing by flat field and correcting atmospheric extinction. Four or more frames were co-added in each filter. They were previously re-centered with a maximum error of 0.1 pix and convolved with a 2D-Gaussian function, in order to correct the seeing differences.

$\overline{1}$ IRAF is distributed by NOAO (National Optical Astronomical Observatories) which are operated by AURA Inc., under contract to the NSF.
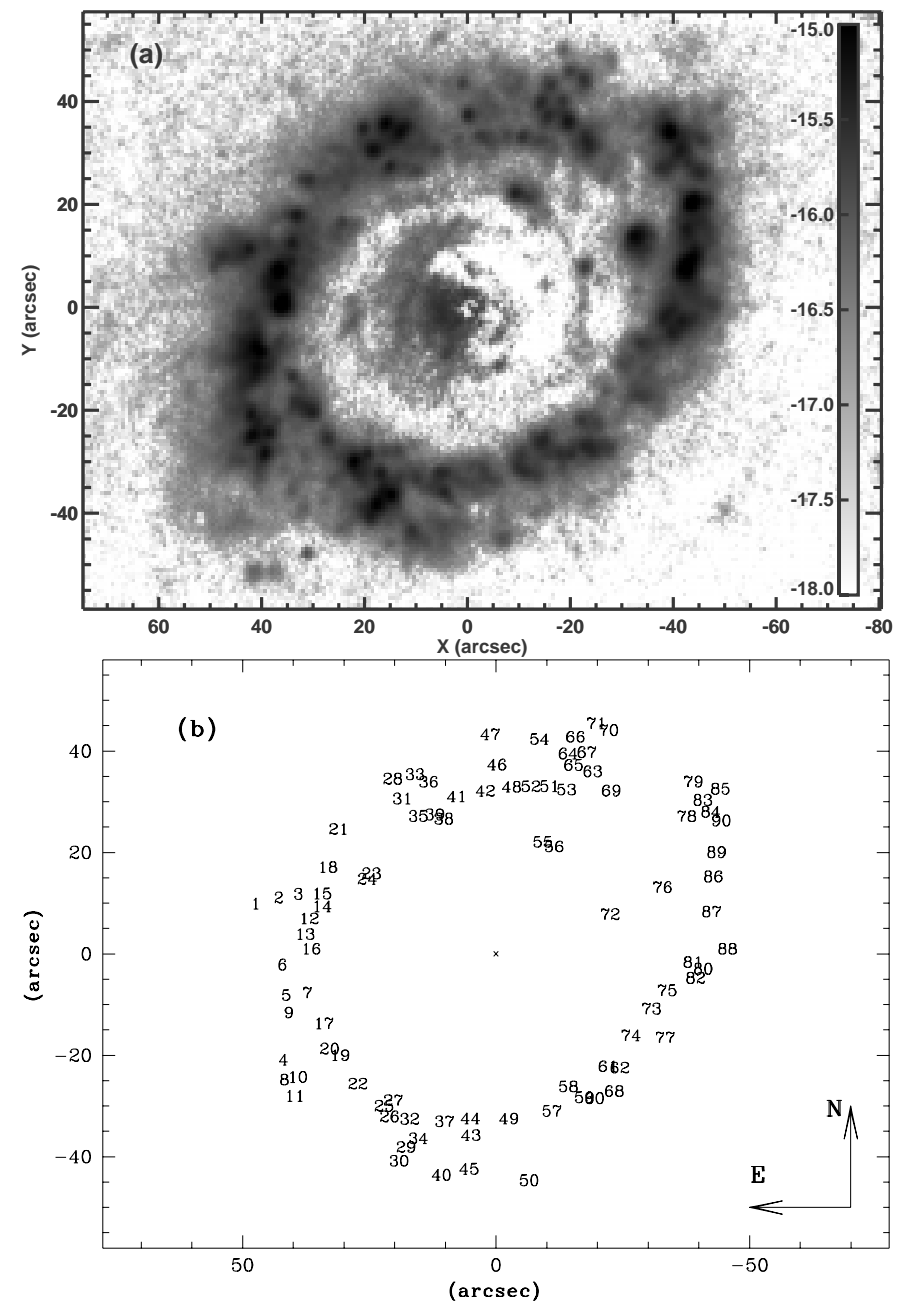

Fig. 1. a) NGC $4736 \mathrm{H} \alpha$ continuum subtracted image in logarithmic grayscale, as indicated by the color bar. b) Identification chart of the NGC 4736 HiI regions

The spectrophotometric standard HD84937 (Oke \& Gunn 1983) was observed to calibrate the final images in absolute flux. This calibration and the subtraction of continuum frames from line plus continuum ones, to map the emission line distribution, were performed following the method outlined by Barth et al. (1994). The sky subtraction is automatically done in this procedure, provided that the sky is similar in both, the line plus continuum and the nearby continuum frames.

The sky background subtraction constitutes a difficult task to study NGC 4736 brightness distribution, since the CCD edges are contaminated by light of the galaxy. In Table 2 we present the sky brightness at each wavelength as measured in the galaxy and standard star frames. In the galaxy frames we averaged four corner areas of $70 \times 70$ pixels and in the standard star frames about half of the frame. Furthermore, in order to improve the $\mathrm{S} / \mathrm{N}$, the background brightness from line plus continuum and continuum frames for each line were also averaged. 
Table 1. Narrow band filters characteristics: Col. 1, ions and continua identification; Cols. 2,3 and 4 give respectively, the filter central wavelength, the FWHM and the maximum transmission; Col. 5, rest wavelength of the transmitted lines; Col. 6, filter transmission at the wavelength of the line corrected by redshift and filter bandpass thermal shift; Col. 7, number of co-added frames; Col. 8, total integration times

\begin{tabular}{lcrcccrr}
\hline Filter & $\begin{array}{c}\lambda_{\text {central }} \\
(\mathrm{A})\end{array}$ & $\begin{array}{c}\text { FWHM } \\
(\mathrm{A})\end{array}$ & $T_{\text {central }}$ & $\begin{array}{c}\lambda_{\text {line }} \\
(\mathrm{A})\end{array}$ & $T_{\text {line }}$ & $N$ & $\begin{array}{r}\text { I. Time } \\
(\mathrm{sec})\end{array}$ \\
\hline$[\mathrm{OII}]$ & 3732 & 52 & 0.42 & $3727 ;-29$ & $0.42 ; 0.42$ & 8 & 12000 \\
{$[\mathrm{OII}] C$} & 3812 & 67 & 0.23 & $\ldots$ & $\ldots$ & 7 & 12000 \\
$\mathrm{H} \beta C$ & 4556 & 114 & 0.52 & $\ldots$ & $\ldots$ & 2 & 2600 \\
$\mathrm{H} \beta$ & 4866 & 52 & 0.63 & 4861 & 0.62 & 7 & 12000 \\
{$[\mathrm{OIII}]$} & 5012 & 50 & 0.62 & 5007 & 0.62 & 7 & 10800 \\
{$[\mathrm{OIII}] C$} & 5276 & 113 & 0.44 & $\ldots$ & $\ldots$ & 2 & 3600 \\
$\mathrm{H} \alpha C$ & 6269 & 132 & 0.52 & $\ldots$ & $\ldots$ & 2 & 3600 \\
$\mathrm{H} \alpha+[\mathrm{NII}]$ & 6569 & 57 & 0.64 & $6563 ;-48 ;-84$ & $0.61 ; 0.60 ; 0.10$ & 6 & 8435 \\
{$[\mathrm{SiI}]$} & 6730 & 48 & 0.64 & $6716 ;-30$ & $0.58 ; 0.62$ & 4 & 7200 \\
{$[\mathrm{SiII}]$} & 9075 & 54 & 0.65 & 9070 & 0.74 & 7 & 10300 \\
{$[\mathrm{SiII}] C$} & 9193 & 145 & 0.35 & $\ldots$ & $\ldots$ & 4 & 6800 \\
\hline
\end{tabular}

The behavior of the background brightness as a function of the wavelength is the same in both data sets, but it is systematically $40 \%$ more intense in the galaxy frames than in the standard star ones. Then, to carry out the sky subtraction in the surface photometry, we preferred the sky background measured in the standard star frames.

We measured the emission lines absolute fluxes of the HiI region of NGC 4736 by aperture photometry (see Sect. 3). The galactic extinction in the zone is $E(B-V)=$ 0.0 (Burnstein \& Heiles 1984). The HiI regions internal reddening was obtained from the decrement of the nebular $\mathrm{H}$ Balmer lines. Balmer emission lines intensity was corrected of the underlying absorption line effect, produced mainly by B, A, and F stars from the ionizing association. This correction has been based on the average value of $1.9 \AA$ of the $\mathrm{H} \alpha$ and $\mathrm{H} \beta$ absorption equivalent width, obtained by McCall et al. (1985) from a sample of 99 Hiı regions in 20 spiral and irregular galaxies. Furthermore, the $\mathrm{H} \alpha$ filter is transparent to the $[\mathrm{NII}]_{\lambda \lambda 6548+6583}$ lines. This contamination has been estimated with $\mathrm{H} \alpha /[\mathrm{NII}]_{\lambda 6583}=3$ relative intensities quoted by Burbidge \& Burbidge (1962) and the filter transmission quoted in Table 1 . The correction amounts to $14 \%$ of the $\mathrm{H} \alpha$ intensity.

The sources of external errors of the observations were analyzed following Barth et al. (1994). The Poissonian noise $\sqrt{N} / N$ for the integrated counts of the most frequent HiI regions is approximately $0.3 \%$ in $\mathrm{H} \alpha, 1.8 \%$ in $\mathrm{H} \beta, 1 \%$ in [OIII] and [SII], and $3.4 \%$ in [OII] and [SIII].

For the atmospheric extinction we adopted the Roque de los Muchachos Observatory's standard curve. The integration times by frame (e.g. $1800 \mathrm{~s}$ ) imply a maximum variation in zenithal angle of $7^{\circ} 30^{\prime}$ during an exposure. Therefore, the use of a mean air mass do not introduces large errors in the photometry.
The differences between the environmental temperature during the observations and that of the laboratory at which the filter transmission curve is measured $\left(20^{\circ} \mathrm{C}\right)$, cause a shift of $0.3 \AA^{\circ} \mathrm{C}^{-1}$ of the curve maximum. This effect has been considered carefully in our reductions, since the instrumental temperature has been registered during the observations.

The statistical fluctuations of the counts in different combined frames also introduce an error. For example, 18 independent observations in $\mathrm{H} \alpha$ of the standard star give a mean of 22286 counts with a standard deviation of $3.7 \%$, after the correction for atmospheric extinction.

A reasonable estimate is that the errors introduced by the above mentioned effects in the absolute flux of a typical HiI region would be about $6 \%$ in [OII], $5 \%$ in $\mathrm{H} \beta$ and [OIII], $4 \%$ in $\mathrm{H} \alpha$ and $6 \%$ in [SII] and [SIII].

\section{The HII regions catalog}

In this section we present the catalog of HiI regions of NGC 4736. Table $3^{a}$ furnishes for each HiI region: Col. 1, the identification number; Col. 2, Hodge \& Kennicutt's (1983) identification; Cols. 3 and 4, the coordinates in arc seconds with respect to the galaxy nucleus, with $X$ toward the East and $Y$ toward the North; Col. 5, the halo diameter; Cols. 6, 7, 8, 9, 10 and 11, [OII $]_{\lambda \lambda 3727+3729}, \mathrm{H} \beta$, $[\mathrm{OIII}]_{\lambda 5007}, \mathrm{H} \alpha,[\mathrm{SII}]_{\lambda \lambda 6716+6730}$, and $[\mathrm{SIII}]_{\lambda \lambda 9070}$, emission line fluxes $\left(\mathrm{erg} \mathrm{cm}^{-2} \mathrm{~s}^{-1}\right)$ respectively; Col. 12, the internal extinction in the $\mathrm{H} \beta$ line; Col. 13 the $\mathrm{H} \alpha$ equivalent width and Col. 14 the $\mathrm{H} \beta$ luminosity. Table $4^{a}$ gives, for the HII regions, the flux of the continuum adjacent to each line.

$\bar{a}$ Tables 3 and 4 are available over the Network in electronic form, via anonymous ftp copy at the CDS: ftp cdsarc.ustrasbg.fr or ftp 130.79.128.5. 

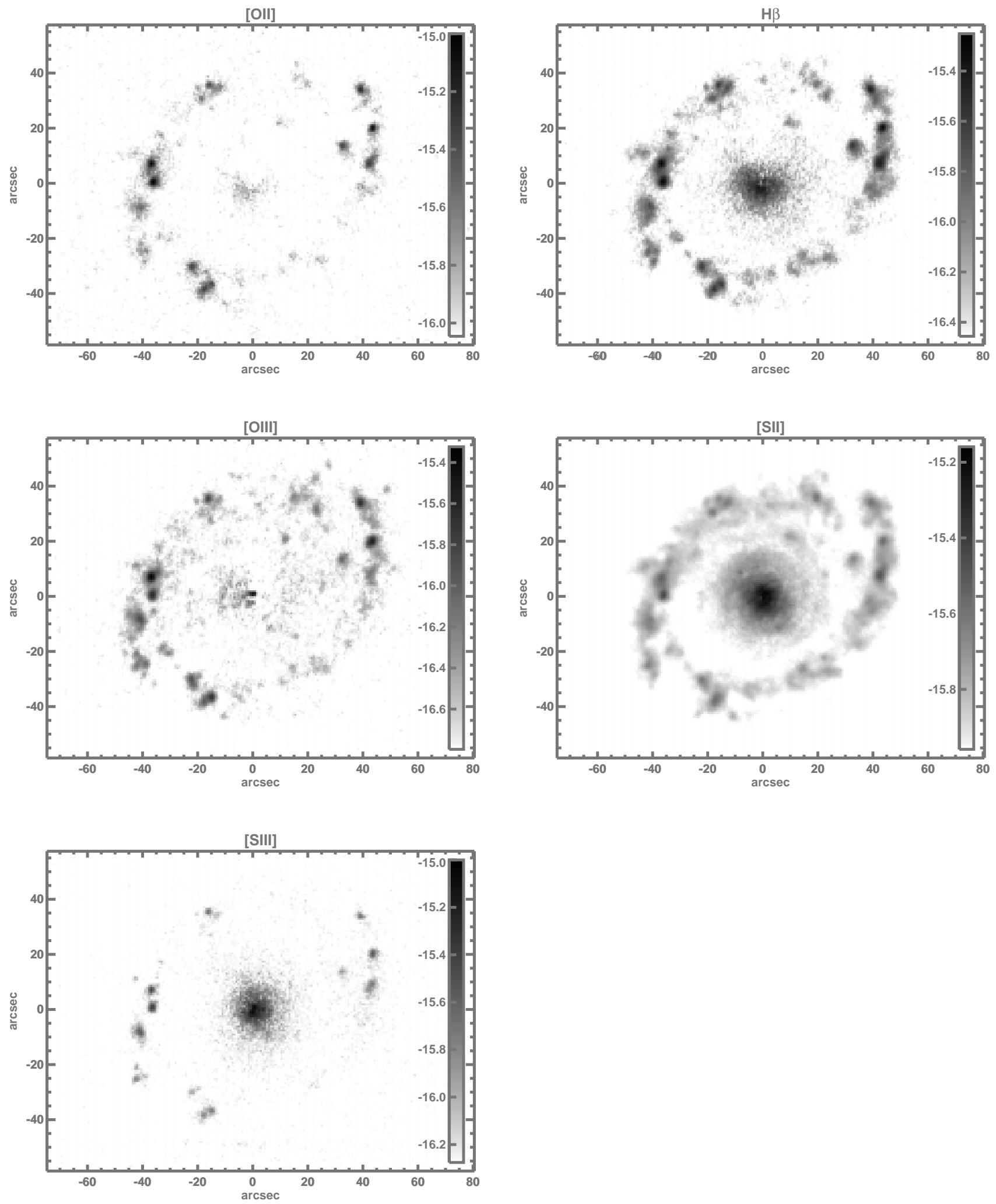

Fig. 2. Pure $[\mathrm{OII}]_{\lambda \lambda 3727+3729}, \mathrm{H} \beta,[\mathrm{OIII}]_{\lambda 5007},[\mathrm{SiI}]_{\lambda 6716+6730}$ and $[\mathrm{SIII}]_{\lambda 9070}$ emission line frames 
Pure line flux and continua frames were deconvolved with the seeing profile in order to measure accurate positions and diameters (we remark that fluxes were not measured in deconvolved frames). This procedure is mainly useful to separate members of crowded groups of HiI regions. The deconvolution was accomplished by means of a Maximum Entropy Method, using astronomical routines of the IDL package. The point spread function (PSF) was obtained through stellar profiles measured in the coadded continuum images. After 100 iterations the deconvolution reduced the FWHM from $\sim 1^{\prime \prime} .8$ to $\sim 00^{\prime \prime} .7$. The method allows to obtain positions with an uncertainty of $\leq 0.1$ pixel.

HII regions diameters were measured in the $\mathrm{H} \alpha$ deconvolved image by isophotal areas, as described in Sect. 4.1.

Figure 1a shows NGC 4736 pure $\mathrm{H} \alpha$ frame and Fig. 1b is an identification chart of the HiI regions. Features that looks like an HII region, whose brightness is below a threshold of three times the background RMS deviation were disregarded.

\section{HII regions physical conditions}

The HII regions of NGC 4736 are mainly distributed in a ring of $3.2 \mathrm{kpc}$ in diameter centered at the nucleus (Fig. 1). A series of short arms detach from the ring outwards. Inwards, we find regions 55, 56 and 72 on a weak $\mathrm{H} \alpha$ emitting arm, which twist itself for more than $180^{\circ}$, from Southwest to East, leaning against the ring to the East. Weak $\mathrm{H} \alpha$ emission is also found in a featureless, hook like structure, surrounding the nucleus to the East, which covers an area of about $1.5 \times 1.0 \mathrm{kpc}^{2}$. Images of the remaining emission lines are presented in Fig. 2.

Table 2. Background brightness at each wavelength as measured in the galaxy and standard star frames. The integration time in Col. 4 corresponds to the data of Col. 3. The integration time for Col. 2 is that quoted in Table 1

\begin{tabular}{llrr}
\hline$\lambda$ & Galaxy & $\begin{array}{c}\text { Std.Star } \\
\AA\end{array}$ & $\begin{array}{r}\text { I. Time } \\
\operatorname{erg} \AA^{-1} \mathrm{~s}^{-1}\left({ }^{\prime \prime}\right)^{-2}\end{array}$ \\
\hline 3812 & $3.00 \mathrm{E}-17$ & $2.06 \mathrm{E}-17$ & 1080 \\
4556 & $2.71 \mathrm{E}-17$ & $2.21 \mathrm{E}-17$ & 608 \\
5276 & $3.67 \mathrm{E}-17$ & $2.29 \mathrm{E}-17$ & 608 \\
6269 & $4.42 \mathrm{E}-17$ & $2.65 \mathrm{E}-17$ & 988 \\
9193 & $2.19 \mathrm{E}-17$ & $1.61 \mathrm{E}-17$ & 1080 \\
\hline
\end{tabular}

\subsection{Diameters distribution}

We have defined an isophotal diameter as $D=$ $\sqrt{\left(D^{\prime}\right)^{2}-\left(D_{\star}^{\prime}\right)^{2}}$, with $D^{\prime}=2 \cdot \sqrt{\frac{A}{\pi}}, D_{\star}^{\prime}=2 \cdot \sqrt{\frac{A_{\star}}{\pi}}$, where $A$ and $A_{\star}$ are the areas inside the isophote of a defined brightness for the HII region and in the half-maximum of

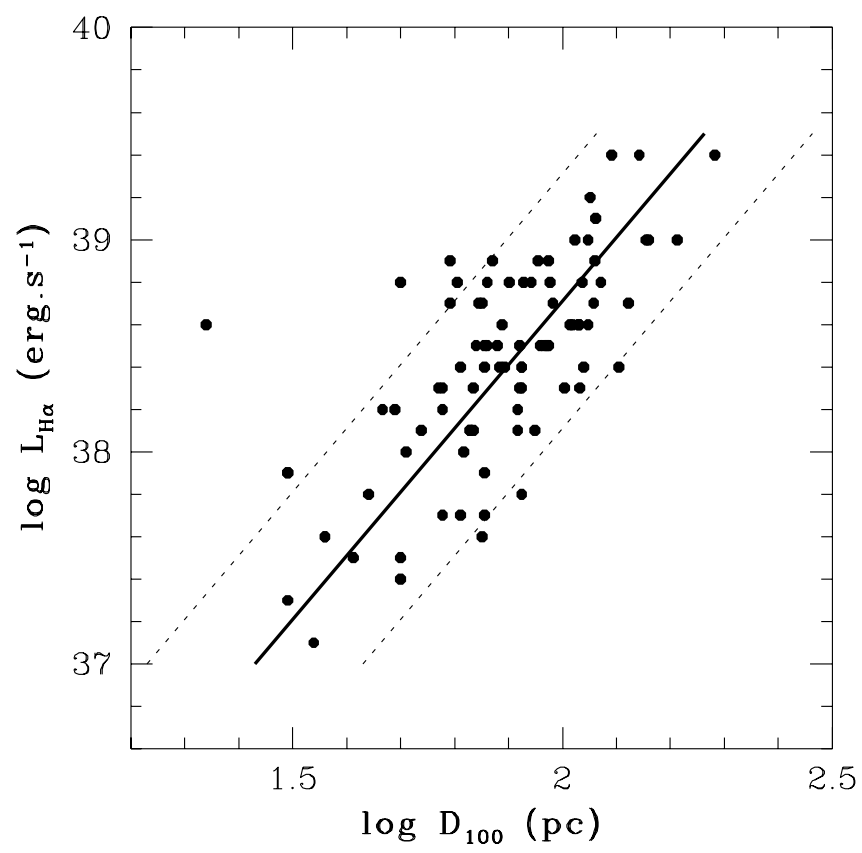

Fig. 3. The lines are $\log L_{\mathrm{H} \alpha}=3 \log D+C$. Full line: $N_{\mathrm{e}}=150 \mathrm{~cm}^{-3}$; dotted lines: extreme values of $N_{\mathrm{e}}\left(500 \mathrm{~cm}^{-3}\right.$ and $40 \mathrm{~cm}^{-3}$ )

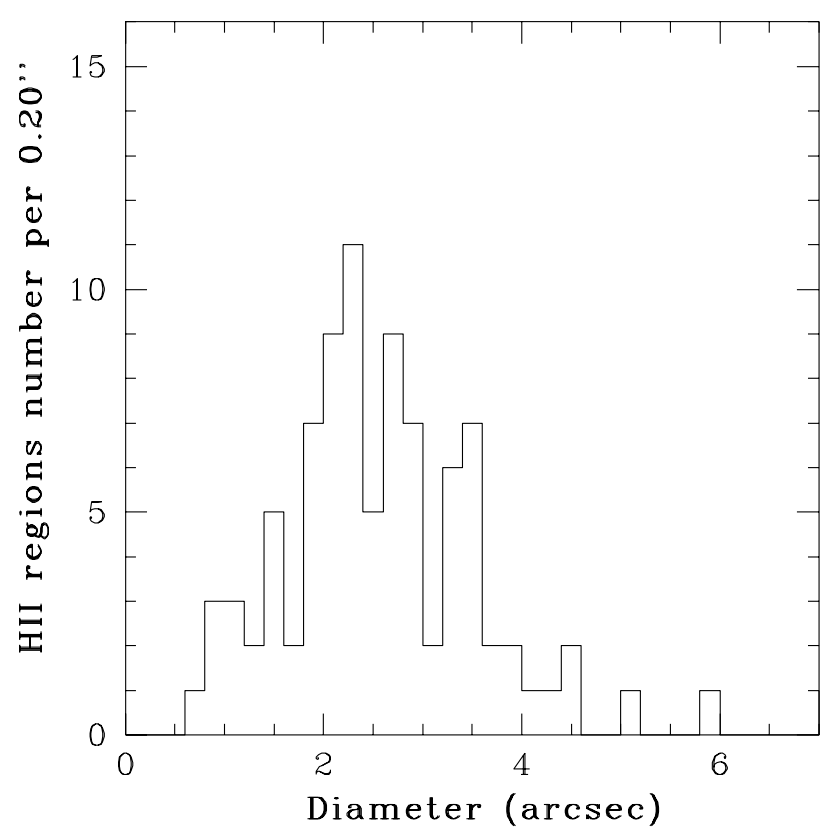

Fig. 4. Numerical diameters distribution for $D_{100}$. The diameter bins are $0^{\prime \prime} 20$ 
a stellar profile respectively. The isophotal diameters have been measured on the deconvolved $\mathrm{H} \alpha$ image (Sect. 3).

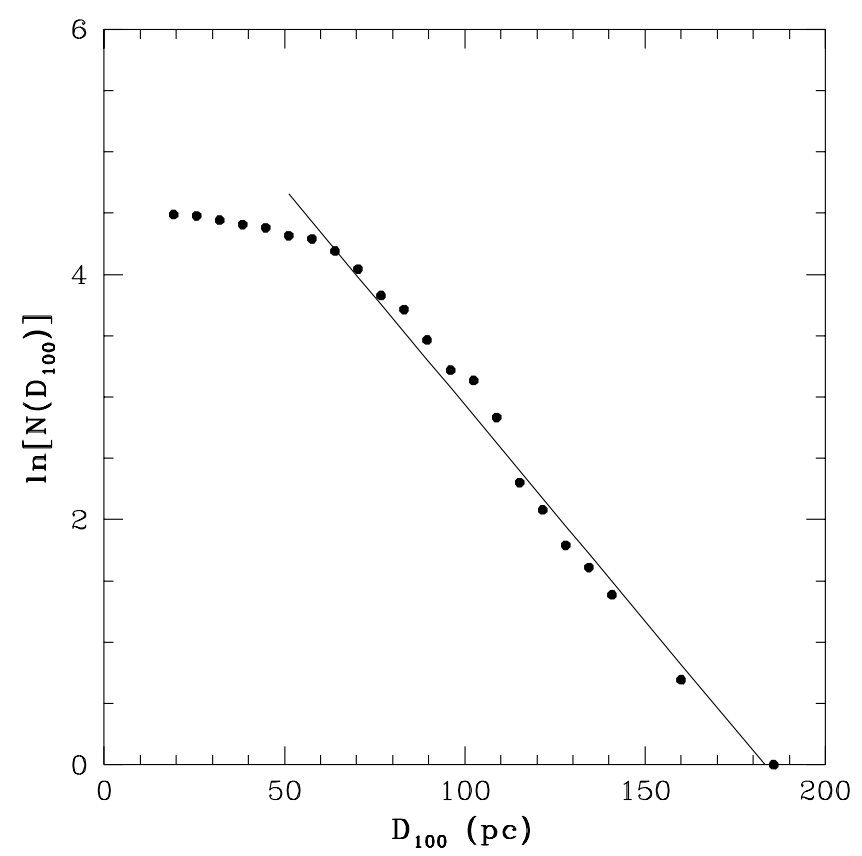

Fig. 5. Cumulative diameters distribution of the Hil regions of NGC 4736. Points shows the number of Hil regions with diameters greater than the value indicated in the horizontal axis. Full line: fit to the law $N(D)=N_{0} \cdot \exp \left(-D / D_{0}\right)$ (see text) with $D_{0}=115 \mathrm{pc}$ and a correlation coefficient of 0.97

We obtained for each HII region a halo diameter $\left(D_{100}\right.$, Kennicut 1988), defined by the isophote at level $2 \times 10^{16} \mathrm{erg} \mathrm{cm}^{-2} \mathrm{~s}^{-1}$, roughly equivalent to the halo diameter used in distance scale applications by Sandage \& Tammann (1974). In Fig. 3 we show a plot of $D_{100}$ vs. $L_{\mathrm{H} \alpha}$. The average slope of the set of points is 3 $\left(\log L_{\mathrm{H} \alpha}=3 \log D+C\right.$, where $C$ is a constant value; full line in the figure), similar to that obtained by Kennicutt (1988) for the brightest HII region of a sample of 95 nearby spiral and irregular galaxies. As quoted by this author, for the simplest case of a constant density ionization-bounded HiI region, the Stromgren sphere should scale as the cube root of the ionizing luminosity. But the average regression in Fig. 3, also represents a locus of constant electron density $N_{\mathrm{e}}$. We show in that figure the lines embracing $95 \%$ of the HII regions which represent the extreme values of $N_{\mathrm{e}}$ in this sample. $N_{\mathrm{e}}$ can be obtained from the following relations:

$$
N_{912}^{0}=\frac{1}{1.428610^{-12}} L_{\mathrm{H} \alpha} \quad\left(T_{\mathrm{e}}=10^{4} \mathrm{~K}\right)
$$

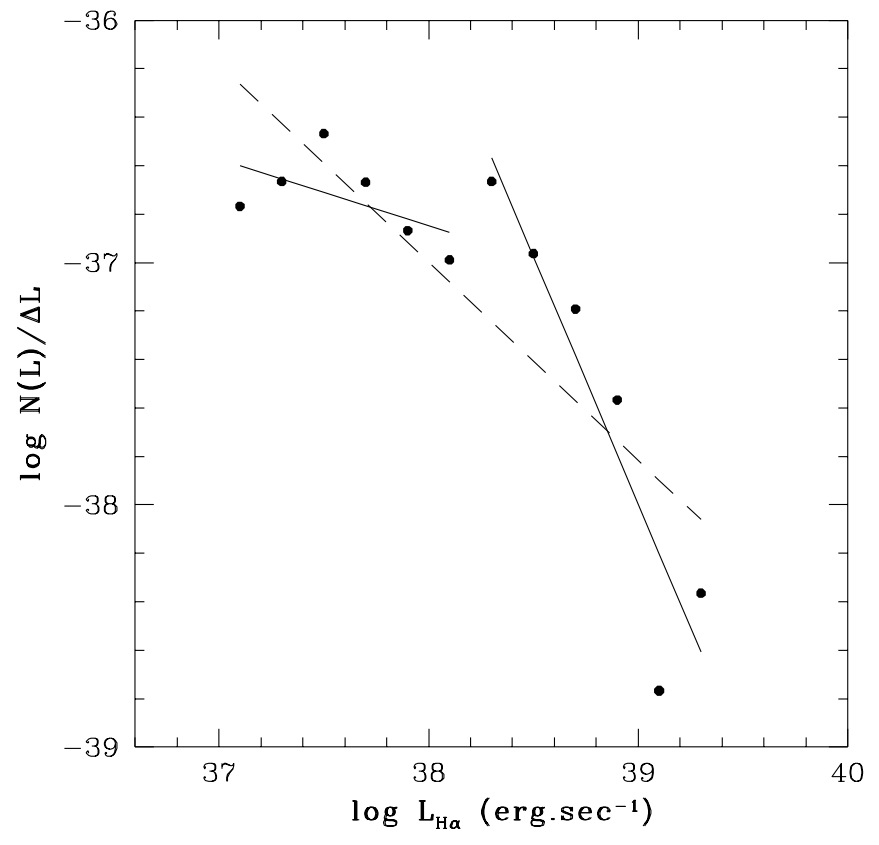

Fig. 6. Differential luminosity function of NGC 4736 HiI regions. Points: average number of HII regions per 0.2 dex luminosity bin. Dashed line gives the fit to the full set of points with the index of the power law $\alpha=0.82$ and the correlation coefficient is -0.85 . Full lines: data set was fitted in two parts. In the low luminosity part we obtained $\alpha=2.02$ and a correlation coefficient of -0.85 ; in the high luminosity part $\alpha=0.27$ whith a correlation correlation coefficient of -0.96

where $N_{912}^{0}$ is the number of photons capable to ionize the Hydrogen. By comparing relation (1) with

$N_{912}^{0}=\frac{4 \pi}{3} r_{1}^{3} N_{\mathrm{e}}^{2} \alpha_{B}$,

where $r_{1}$ is the Strömgren radius, $\alpha_{B}$ is the recombination coefficient to all excited levels, and $N_{\mathrm{e}}=N_{\mathrm{H}}$, the hydrogen atoms number density (Osterbrock 1989). We obtained for the average and the two extreme $N_{\mathrm{e}}$ values, $150 \mathrm{~cm}^{-3}, 500 \mathrm{~cm}^{-3}$ and $40 \mathrm{~cm}^{-3}$ respectively. As a matter of comparison, from Kennicutt's (1988, Fig. 6.) data we calculated $20 \mathrm{~cm}^{-3}, 200 \mathrm{~cm}^{-3}$ and $4 \mathrm{~cm}^{-3}$ respectively. For the three regions in common with Kennicutt (regions 12, 16 and 87 in our sample), his mean $N_{\mathrm{e}}$ is $70 \mathrm{~cm}^{-3}$, while our one is $200 \mathrm{~cm}^{-3}$. The difference appear mainly from the measured diameters. For these regions, Kennicutt's mean diameter is $\log D_{100}(\mathrm{pc})=2.5$, while we obtained $\log D_{100}(\mathrm{pc})=2.2$. The deconvolution process might explain such a difference.

Figure 4 shows $D_{100}$ histogram. The distribution of halo diameters is fairly symmetric and peaks at $D_{100} \approx$ 2 ". 3 .

Figure 5 shows the cumulative distribution of $D_{100}$. It is fitted by the law $N(D)=N_{0} \cdot \exp \left(-D / D_{0}\right)$ (e.g. van den Bergh 1981; Hodge 1983, 1987), where $N(D)$ represents the number of HII regions with a diameter larger 

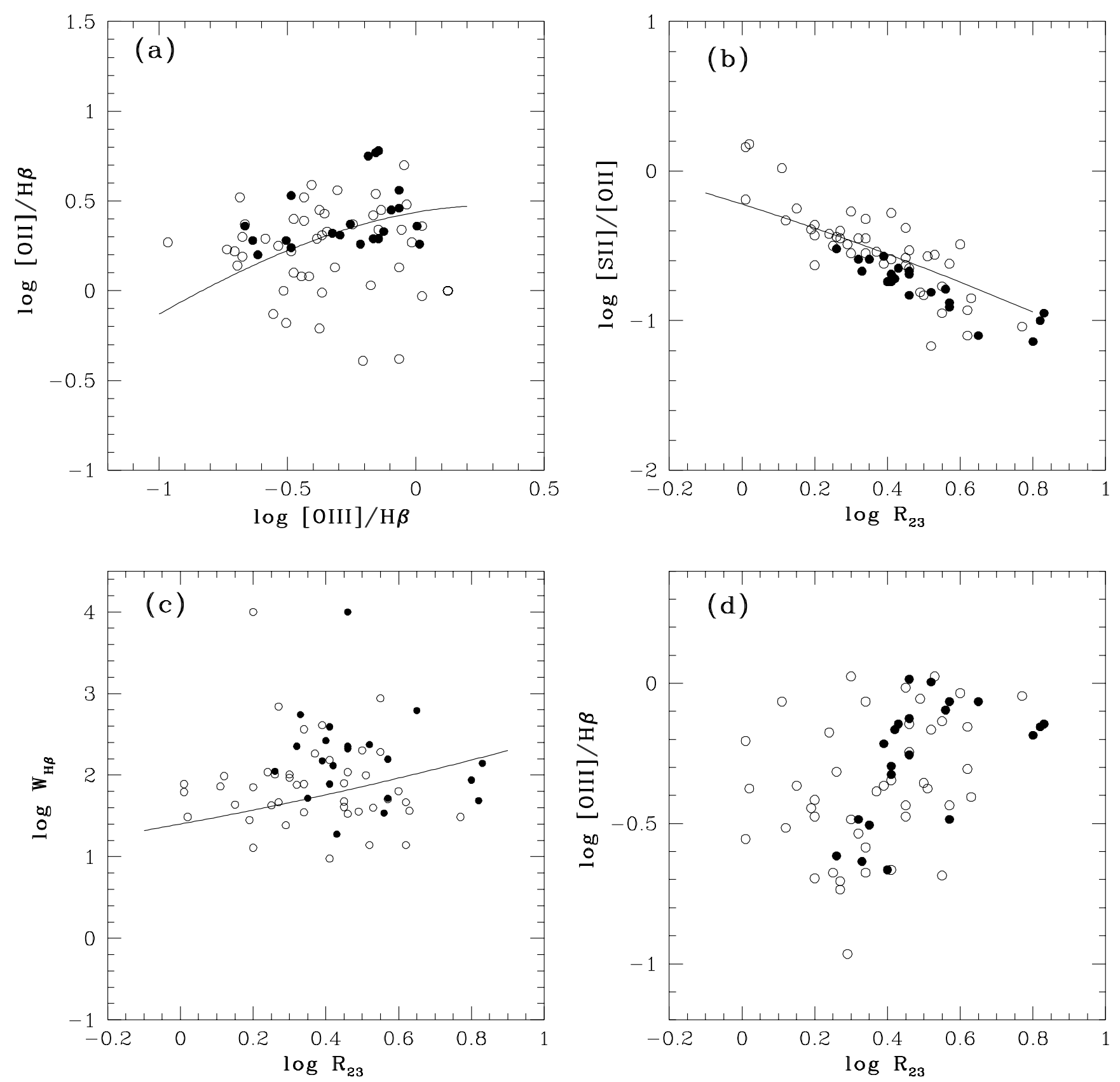

Fig. 7. Line ratio pairs relations. a) $\log [\mathrm{OII}] / \mathrm{H} \beta$ vs. $\log [\mathrm{OIII}] / \mathrm{H} \beta$; b) $\log [\mathrm{SII}] /[\mathrm{OII}]$ vs. $\left.\log R_{23} ; \mathbf{c}\right) \log W_{\mathrm{H} \beta}$ vs. $\left.\log R_{23} ; \mathbf{d}\right)$ $\log [\mathrm{OIII}] / \mathrm{H} \beta$ vs. $\log R_{23}$. Full lines in $\left.\mathbf{a}\right)$, b) and $\mathbf{c}$ ) the MRS's predicting equations. Black dots represents Hil regions with $\log L_{\mathrm{H} \alpha} \geq 38.8\left(\mathrm{erg} \mathrm{s}^{-1}\right)$

than a characteristic diameter $D$. A least square fit gives $D_{0}=115 \mathrm{pc}$, with a correlation coefficient of 0.97 and it is represented by the full line in Fig. 5 .

As a matter of comparison, for $N_{\mathrm{e}} \approx 100 \mathrm{~cm}^{-3}, D_{0}$ is approximately five times larger than the radius of the Strömgren's sphere of an O5 star, indicating that the largest HII regions are certainly ionized by associations of massive stars. The total number of HII regions furnished by the regression is $N_{0} \approx 600$. The fall down of $N(D)$ for $D_{100} \lesssim 50$ pc with respect to the quoted regression indi- cates that we detected only 2 to $5 \%$ of the weakest HII regions.

\subsection{Luminosity function}

HII regions luminosity functions are generally well represented by power laws $\mathrm{d} N(L)=A L^{-\alpha} \mathrm{d} L$, where $\mathrm{d} N(L)$ is the number of HiI regions emitting an $\mathrm{H} \alpha$ luminosity between $L$ and $L+\mathrm{d} L$. In Fig. 6 we show the differential luminosity function for the HII regions of NGC 4736, where the 
Table 5. HiI regions oxygen abundance $12+\log (\mathrm{O} / \mathrm{H})$ and temperature index $\eta$

\begin{tabular}{|c|c|c|c|c|c|}
\hline Reg & $12+\log \left(\frac{\mathrm{O}}{\mathrm{H}}\right)$ & $\eta$ & Reg & $12+\log \left(\frac{\mathrm{O}}{\mathrm{H}}\right)$ & $\eta$ \\
\hline 2 & 9.03 & 0.88 & 49 & 9.26 & $\ldots$ \\
\hline 4 & 9.06 & 1.06 & 51 & 9.04 & 1.30 \\
\hline 5 & 8.85 & 1.59 & 55 & 9.04 & $\ldots$ \\
\hline 7 & 8.63 & $\ldots$ & 56 & 8.98 & $\ldots$ \\
\hline 8 & 9.09 & 1.13 & 57 & 9.17 & 1.56 \\
\hline 9 & 9.07 & 1.10 & 58 & 8.94 & $\ldots$ \\
\hline 10 & 8.93 & 1.24 & 59 & 9.19 & $\ldots$ \\
\hline 11 & 9.12 & 1.29 & 60 & 8.96 & $\ldots$ \\
\hline 12 & 9.04 & 1.13 & 61 & 8.58 & 0.98 \\
\hline 13 & 9.10 & 1.21 & 62 & 9.19 & 1.13 \\
\hline 14 & 9.14 & 0.70 & 63 & 9.11 & 0.85 \\
\hline 15 & 9.21 & 0.65 & 64 & 8.87 & 1.08 \\
\hline 16 & 9.07 & 1.45 & 65 & 8.68 & 1.11 \\
\hline 18 & 8.94 & 1.37 & 66 & 9.11 & 1.44 \\
\hline 19 & 9.17 & 0.85 & 68 & 9.14 & 1.61 \\
\hline 20 & 8.95 & $\ldots$ & 69 & 9.26 & $\ldots$ \\
\hline 23 & 8.99 & 1.22 & 70 & 9.11 & 0.72 \\
\hline 25 & 9.03 & 1.09 & 75 & 9.16 & $\ldots$ \\
\hline 26 & 9.04 & 0.43 & 76 & 9.13 & 1.33 \\
\hline 27 & 9.16 & 1.19 & 77 & 9.07 & $\ldots$ \\
\hline 28 & 8.89 & 1.49 & 79 & 9.06 & 1.13 \\
\hline 29 & 9.03 & 1.28 & 80 & 9.01 & 1.21 \\
\hline 30 & 9.14 & 1.44 & 81 & 9.15 & 0.95 \\
\hline 31 & 9.07 & 1.14 & 82 & 9.08 & 1.02 \\
\hline 33 & 9.01 & 1.39 & 83 & 9.22 & 0.76 \\
\hline 34 & 8.99 & 1.20 & 84 & 9.23 & $\ldots$ \\
\hline 35 & 8.98 & 1.65 & 85 & 9.26 & $\ldots$ \\
\hline 36 & 9.11 & 1.33 & 86 & 9.08 & 0.99 \\
\hline 40 & 8.91 & $\ldots$ & 87 & 9.16 & 1.28 \\
\hline 41 & 8.88 & $\ldots$ & 88 & 9.19 & $\ldots$ \\
\hline 43 & 8.60 & $\ldots$ & 89 & 9.03 & 1.28 \\
\hline 44 & 9.13 & 1.31 & 90 & 8.96 & $\ldots$ \\
\hline 46 & 9.19 & $\ldots$ & & & \\
\hline
\end{tabular}

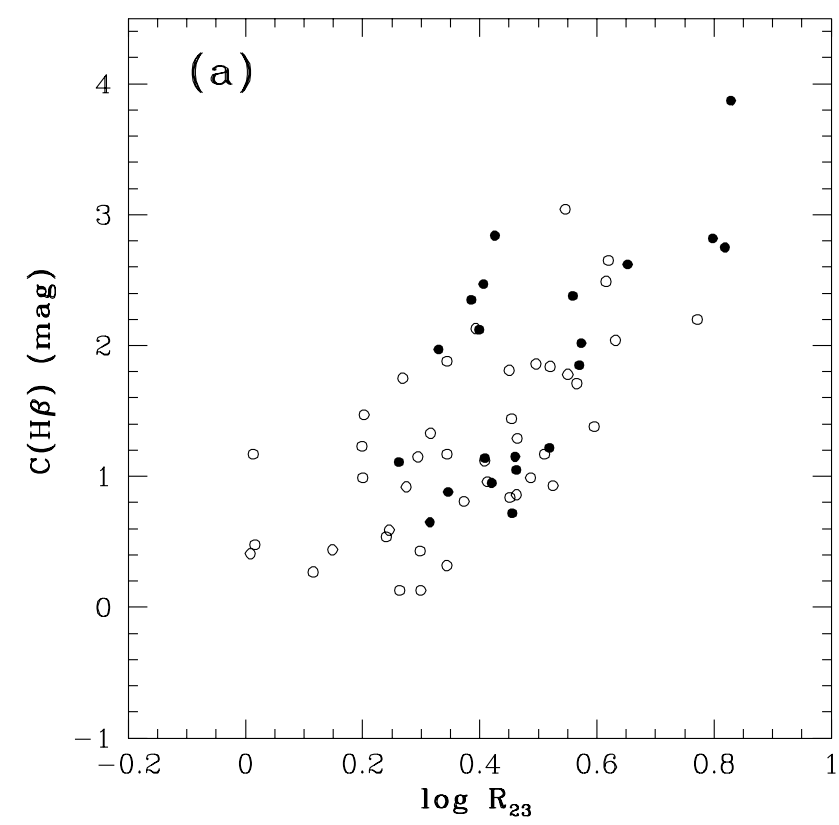

Fig. 8. HiI regions internal extinction $C(\mathrm{H} \beta)$ vs. $R_{23} \equiv([\mathrm{OII}]+[\mathrm{OIII}]) / \mathrm{H} \beta$. Black dots represents HII regions with $\log L_{\mathrm{H} \alpha} \geq 38.8\left(\mathrm{erg} \mathrm{s}^{-1}\right)$ points are the average number of HII regions per 0.2 dex luminosity bin. The general properties coincide with that obtained by Kennicutt et al. (1989). Basically, it deviates strongly from a single power law, as happens with HiI regions in early-type spirals. NGC 4736, as M 51, NGC 3521 and NGC 3627, presents a turn over in the LF for HII regions brighter than $\log L_{\mathrm{H} \alpha}=38.7-39.0\left(\operatorname{erg~s}^{-1}\right)$, a signature of late-type spirals and irregulars. The number of this type of HII region is lower than that which would correspond to the extrapolation of the power law representing the luminosity function of the faintest HII regions. A discussion about the implications of this property can be found in Kennicutt et al. (1989).

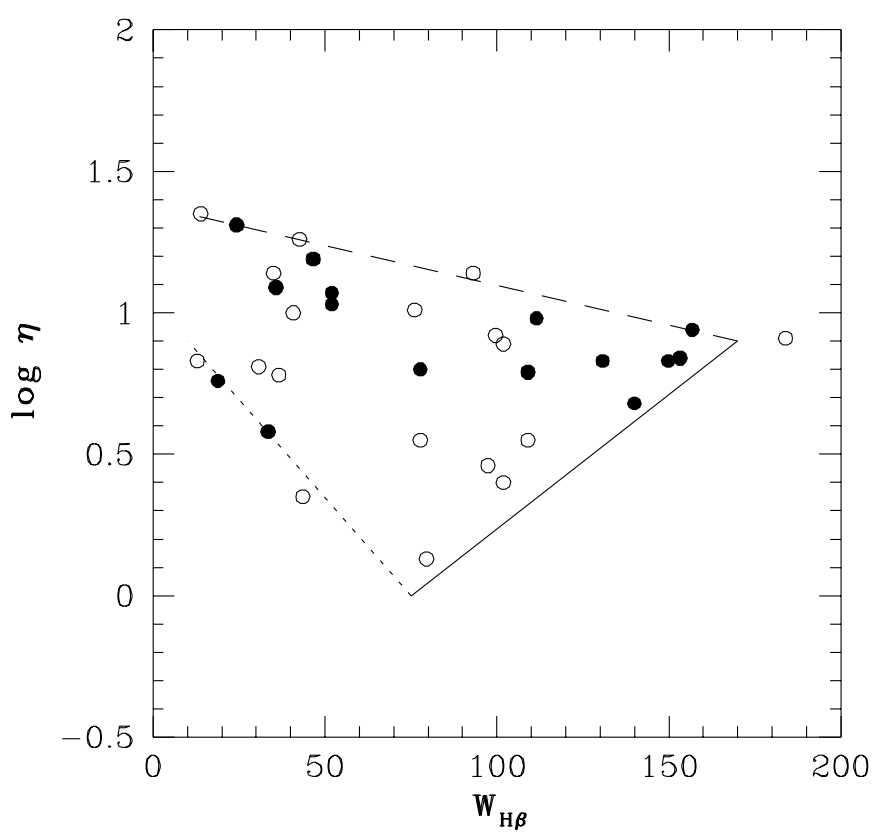

Fig. 9. Relation of the temperature parameter $\eta$ with $\mathrm{H} \beta$ equivalent width

\subsection{Chemical abundances}

The Oxygen chemical abundance, $12+\log \left(\frac{\mathrm{O}}{\mathrm{H}}\right)$, was estimated by means of the ratio $R_{23}=([\mathrm{OII}]+[\mathrm{OIII}]) / \mathrm{H} \beta$ (Pagel et al. 1979; McCall et al. 1985, hereafter MRS), calibrated according to Zaritsky et al. (1994):

$$
\begin{aligned}
12+\log \left(\frac{\mathrm{O}}{\mathrm{H}}\right)= & 9.265-0.33 X-0.202 X^{2} \\
& -0.207 X^{3}-0.333 X^{4},
\end{aligned}
$$

where $X=\log R_{23}$. Since we observed only [OIII $]_{\lambda 5007}$, the total intensity was obtained from $[\mathrm{OIII}]_{\lambda \lambda 4959+5007}=$ $1.335 \times[\mathrm{OIII}]_{\lambda 5007}$. Table 5 gives the set of derived Oxygen abundances, whose mean from 65 HiI regions is $12+$ $\log \left(\frac{\mathrm{O}}{\mathrm{H}}\right)=9.05$ with $\sigma=0.15$. Since the HII regions are 


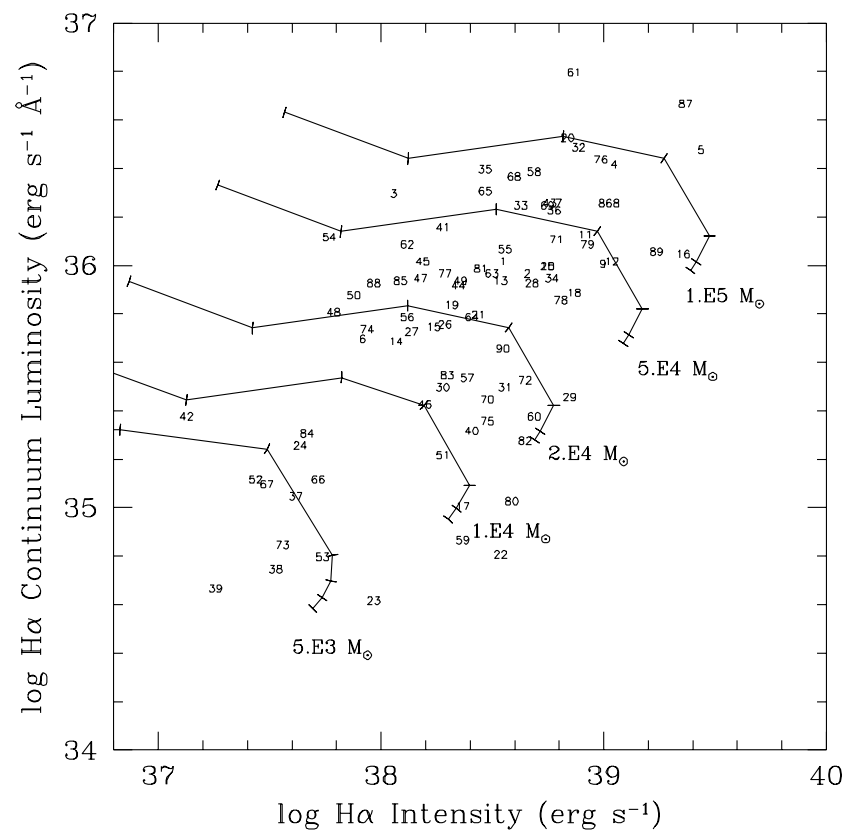

Fig. 10. $\mathrm{H} \alpha$-continuum luminosity vs. $\mathrm{H} \alpha$ intensity compared to the evolutionary tracks of theoretical ionizing clusters (full lines). The models are: $M_{\text {tot }}=1.0 E 5 M_{\odot}, M_{\text {up }}=40 M_{\odot}$; $M_{\mathrm{tot}}=5.0 E 4 M_{\odot}, M_{\mathrm{up}}=40 M_{\odot} ; M_{\mathrm{tot}}=2.0 E 4 M_{\odot}$, $M_{\mathrm{up}}=39 M_{\odot} ; M_{\mathrm{tot}}=1.0 E 4 M_{\odot}, M_{\mathrm{up}}=37 M_{\odot} ;$ $M_{\text {tot }}=5.0 E 3 M_{\odot}, M_{\text {up }}=28 M_{\odot}$. The tick marks on each model represent the model runs from right to left with $t=0$, $1,3,5,7,9$ and $12 \mathrm{Myr}$

practically located at the same radial distance, we can not prove radial metallicity gradients.

In Fig. 7 we show MRS diagnostic diagrams, $\log [\mathrm{OII}] / \mathrm{H} \beta$ vs. $\log [\mathrm{OIII}] / \mathrm{H} \beta, \log [\mathrm{SII}] /[\mathrm{OII}] \quad$ vs. $\log R_{23}$, $\log W_{\mathrm{H} \beta}$ vs. $\log R_{23}, \log [\mathrm{OIII}] / \mathrm{H} \beta$ vs. $\log R_{23}$, where we plot our data set and MRS's predicting equations. Except for $\log W_{\mathrm{H} \beta}$ vs. $\log R_{23}$, the positions of the HII regions brighter than $\log L_{\mathrm{H} \alpha}=38.8\left(\mathrm{erg} \mathrm{s}^{-1}\right)$ are in good agreement with MRS's predicting equations.

In Fig. 8 we plot the HII regions internal extinction $C(\mathrm{H} \beta)$ vs. $R_{23}$, which shows that higher extinction corresponds with lower metallicities. A possible reason for the observed correlation is that, on average, the higher metallicity, the stronger are the stellar winds and consequently they are more efficient in blowing out and breaking the dust grains within the HII region. We point out that this correlation cannot be a consequence of the [OII] extinction correction, since $R_{23}$ is more influenced by [OIII] $/ \mathrm{H} \beta$ as can be seen in Figs. $7 \mathrm{a}$ and $7 \mathrm{~d}$.

\subsection{Temperatures of the ionizing sources}

The effective temperature of the HII regions ionizing source $\left(T_{\star}\right)$ can be estimated through the parameter $\eta=$
$\left(\mathrm{O}^{+} / \mathrm{O}^{++}\right) /\left(\mathrm{S}^{+} / \mathrm{S}^{++}\right)$(Vílchez \& Pagel 1988), which can be obtained from the observed line ratio

$\eta^{\prime}=\left(\frac{[\mathrm{OII}]_{\lambda \lambda 3727+3729}}{[\mathrm{OIII}]_{\lambda \lambda 4959+5007}}\right) /\left(\frac{[\mathrm{SII}]_{\lambda \lambda 6716+6739}}{[\mathrm{SIII}]_{\lambda \lambda 9070+9532}}\right)$,

as

$\log \eta=\log \eta \prime+\frac{0.14}{t}+0.16$

where $t$ is the electronic temperature $T_{\mathrm{e}}$ in units of $10^{4} \mathrm{~K}$. $\eta$ is a good criterion of effective temperature of ionizing stars $\left(T_{\star}\right)$ and it is relatively insensitive to chemical composition and ionization conditions found in observed nebulae, particularly when measurements of the [SIII] lines in the far red are available, as happens in our case. Assuming $t=1$, we can compute $[\mathrm{SIII}]_{\lambda \lambda 9532+9070}=3.48[\mathrm{SIII}]_{\lambda 9070}$ (Mendoza \& Zeippen 1982). Table 5 gives the values of $\eta$ for 45 HiI regions.

Figure 9 gives the distribution of the HII regions in the plane $\log \eta$ vs. $W_{\mathrm{H} \beta}$. If the formation of stars in the ionizing cluster occurs in a burst, the evolution of the HII regions as a function of time in this diagram will be in the sense of decreasing $W_{\mathrm{H} \beta}$ and increasing $\eta$ (decreasing $T_{\star}$ ) (Copetti et al. 1986, CPD). The full line in Fig. 9 limits the highest $W_{\mathrm{H} \beta}$ values. It is the locus of the youngest HII regions for a given $\eta$, according to CPD. As a consequence, the upper envelope of the data points (dashed line) would represent the evolutionary track or fading of the most luminous HiI regions (black dots, $\log L_{\mathrm{H} \alpha} \geq 38.7$ ). The lower envelope (dotted line) shows a higher slope than the upper one, indicating that the less massive Hit regions suffer more drastic change in temperature during their fading. In the next section we carry out a quantitative analysis of the Hir regions evolution.

\subsection{Age and mass of the ionizing clusters}

We discuss the HiI region distribution in the plane $\mathrm{H} \alpha$ intensity vs. $\mathrm{H} \alpha$-continuum luminosity, which is very suitable to study the ionizing clusters properties, because the $\mathrm{H} \alpha$ intensity measures the amount of ionizing photons furnished directly by the more massive stars in the Main Sequence, while the $\mathrm{H} \alpha$-continuum luminosity is mainly contributed by low-mass stars and evolved massive stars. In this context, we derive age and mass of the HII region ionizing clusters. The data set is compared to theoretical models of the ionizing clusters, which are computed with the "ET" code (Cid-Fernandes et al. 1992), which retrives the temporal evolution of a cluster spectrum by inputting (i) An IMF formed in a burst, (ii) Stellar evolutionary tracks and (iii) Model atmospheres, besides the cluster total mass $M_{\mathrm{T}}$. We assume as input data for "ET": (i) A Salpeter's IMF with a lower mass limit $M_{\text {low }}=1 M_{\odot}$ and upper mass limit $M_{\text {up }}=40 M_{\odot}$, for clusters with total mass $M_{\mathrm{T}} \geq 210^{4} M_{\odot} ; M_{\text {up }}=37 M_{\odot}$, for those with $M_{\mathrm{T}}=10^{4} M_{\odot}$; and $M_{\text {up }}=28 M_{\odot}$, for those with 

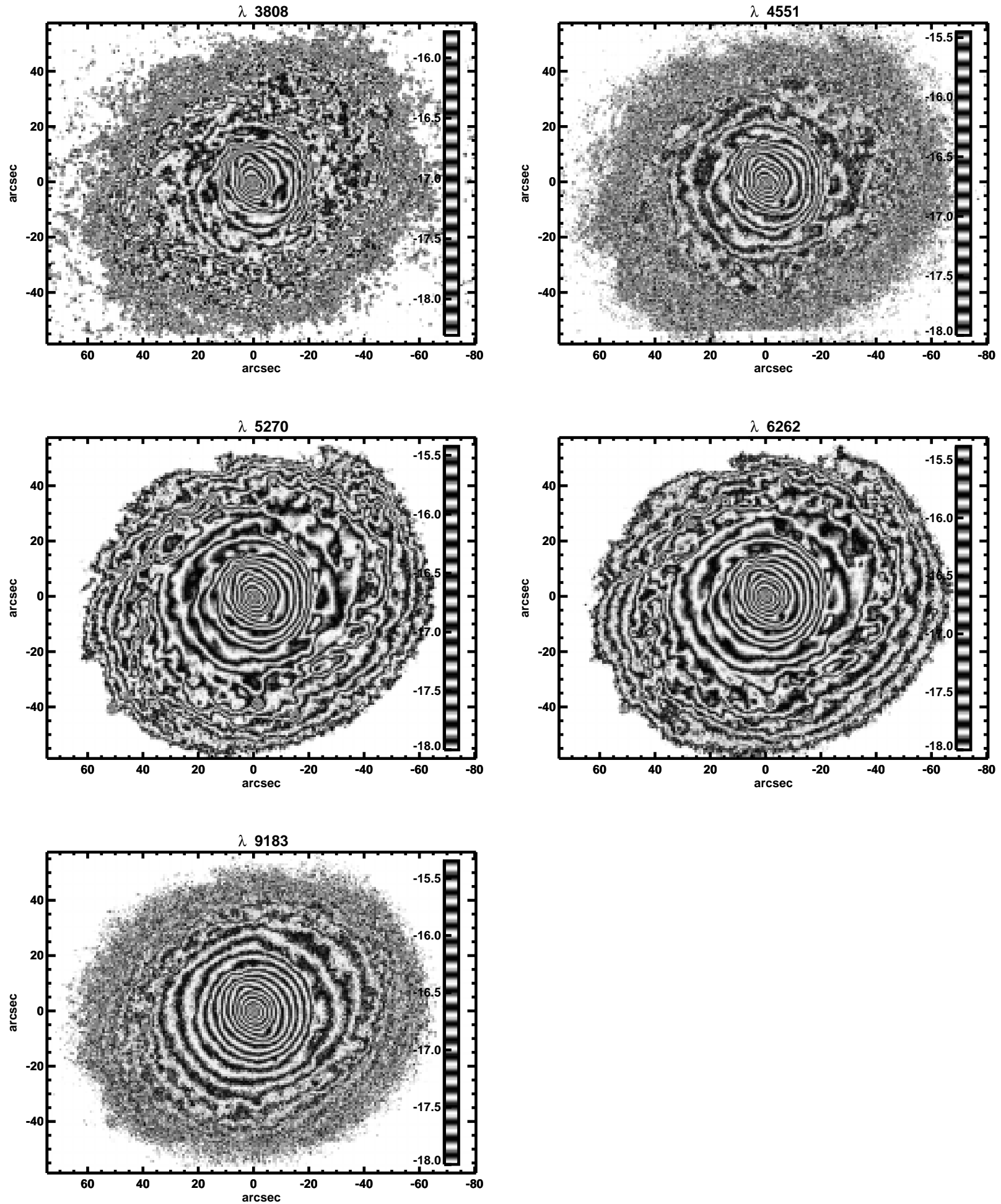

Fig. 11. Continua images at $\lambda 3808 \AA, \lambda 4551 \AA, \lambda 5270 \AA, \lambda 6262 \AA$ and $\lambda 9183 \AA$. North is up, East is to the left. A saw-tooth lookup table was used; levels in the color bars are logarithm of flux density (erg $\AA^{-1} \mathrm{~cm}^{-2} \mathrm{~s}^{-1} \mathrm{pix}^{-1}$ ) 

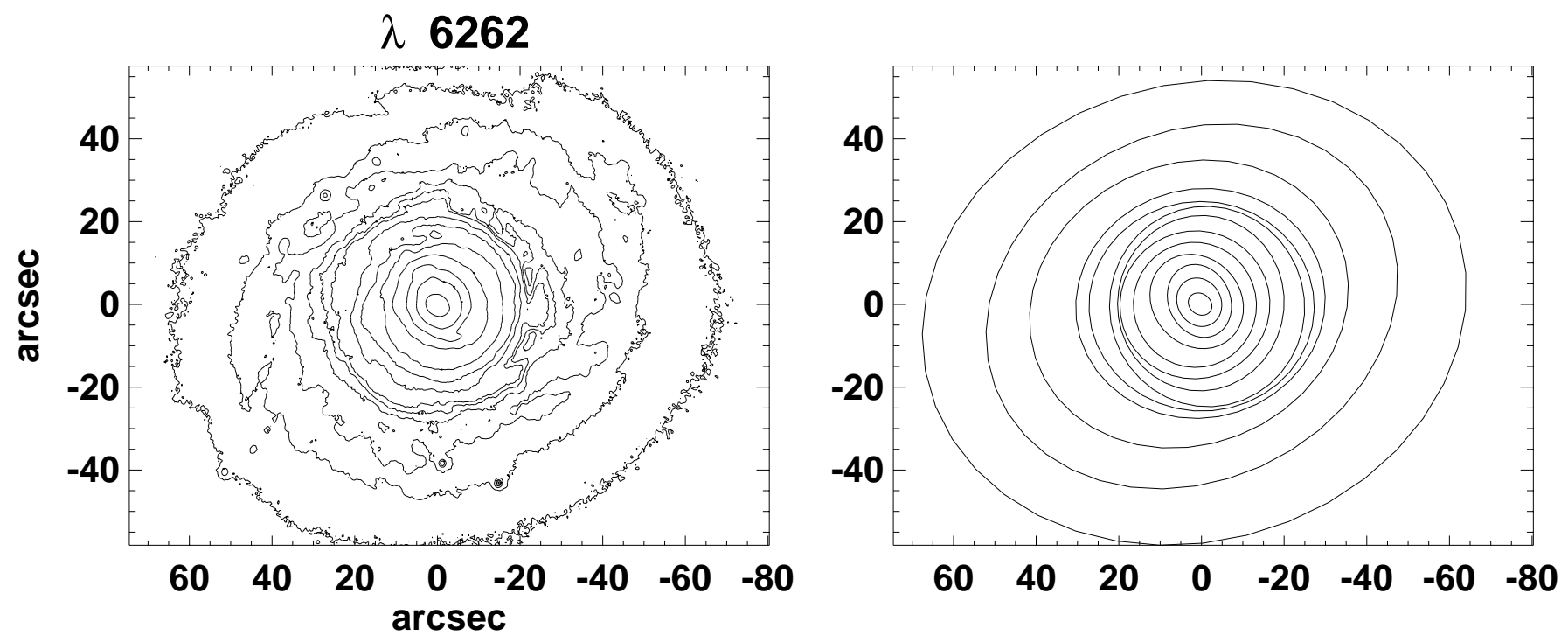

Fig. 12. Result of the isophote fits to the $\lambda 6262 \AA$ image. Left pannel: the isophotal map; brightness levels are 15.4, 16.0, 16.6, 17.0, 17.6, 18.0, 18.2, 18.5, 18.6, 18.8, 19.2, 19.8, and 21.0 mag arcsec ${ }^{-2}$. Right pannel: fitting for the same levels, which corresponds to ellipses with semi-major axis length of 10, 20,30,40,50,60,70, 80,90, 100, 130, 170 and 220 arcsec
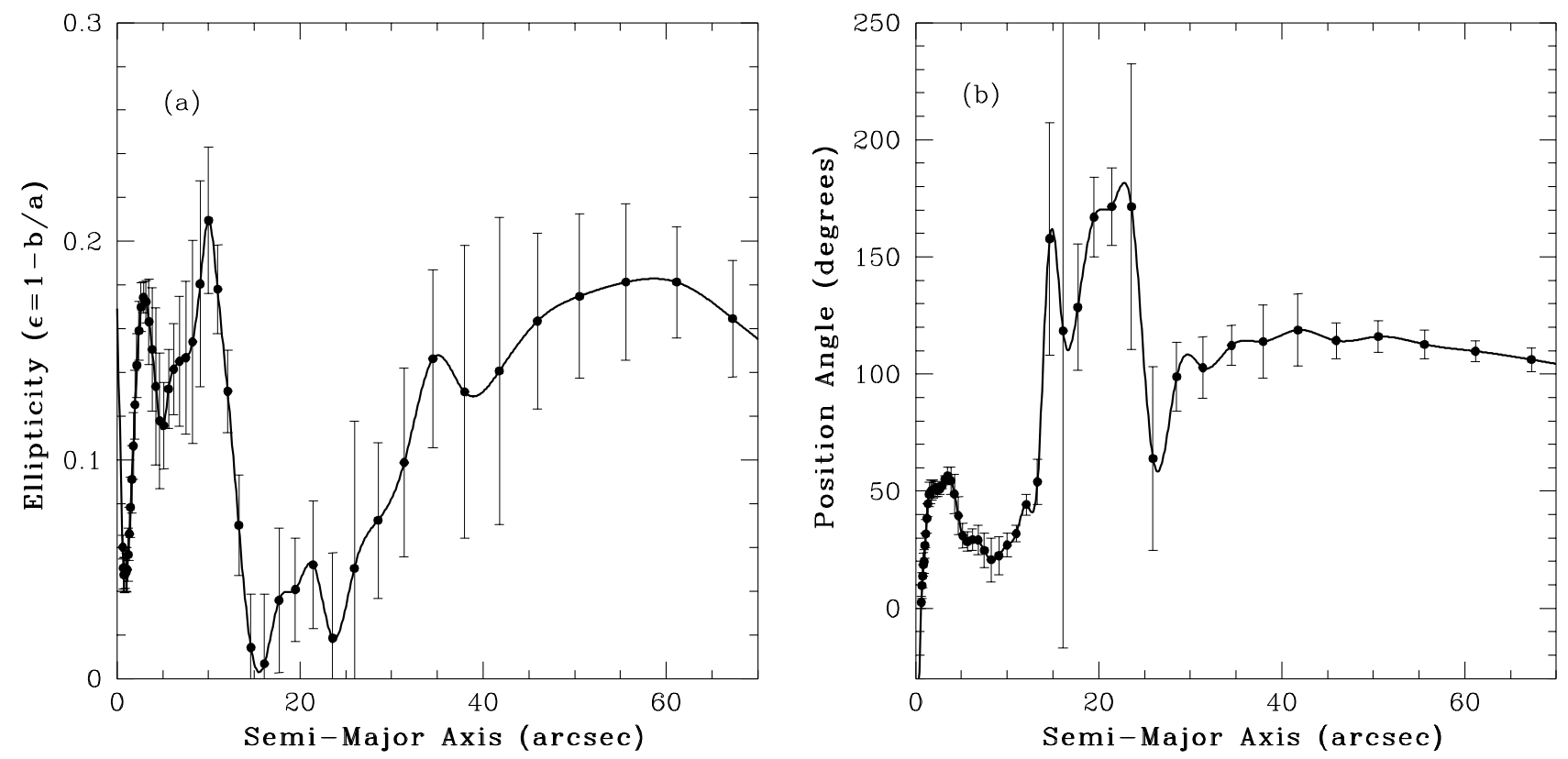

Fig. 13. Ellipticities and position angles vs. distance along the semi-major axis in $\lambda 6262 \AA$ image. Error bars are obtained from the residual intensity scatter, combined with the internal error in the harmonic fit, after removal of the first and second fitted harmonics

$M_{\mathrm{T}}=510^{3} M_{\odot}$, suitable for the range of sources temperature derived in the previous section. Particularly, for the two last cases, a larger $M_{\text {up }}$ would require a fractional number of most massive star. (ii) The set of stellar evolutionary tracks of Maeder \& Meynet (1988). (iii) The model atmospheres of Kurucz (1979). For a theoretical cluster of a given mass, "ET" furnishes, beside other data, the $\mathrm{H} \alpha$ continuum luminosity and the $\mathrm{H} \alpha$-line intensity, which are computed by assuming a radiation bounded HiI region (case B of Backer \& Mentzel 1936).

In Fig. 10 we have plotted the data set in the plane $\mathrm{H} \alpha$-continuum luminosity vs. $\mathrm{H} \alpha$ intensity, where we have superimposed the evolutionary tracks of cluster models, up to 12 Myr. It becomes clear that the right-hand side limit of the data set distribution corresponds to a Zero Age Sequence for clusters of different masses. The more massive ionizing clusters are those of regions 5, 51 and 

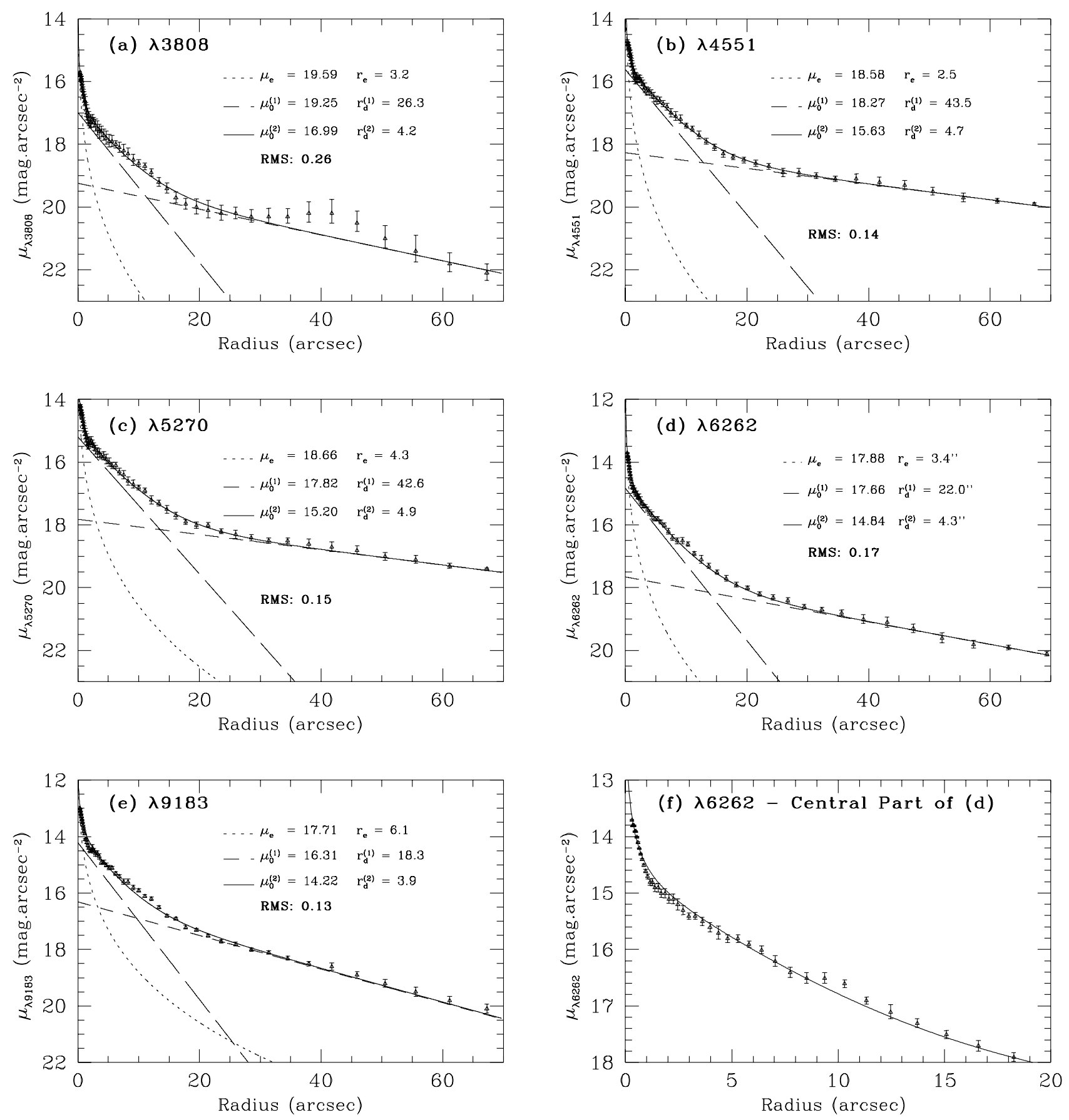

Fig. 14. Radial brightness profiles (data points) and its best fits (full lines) for $\lambda 3808$ a), $\lambda 4551 \mathbf{b}$ ), $\lambda 5270 \mathbf{c}$ ), $\lambda 6262 \mathbf{d}$ ) and $\lambda 9183$ e). Individual components of each fit are shown separatelly: de Vaucouleurs' profiles in dotted lines; Freeman profiles in dashed lines. We give the values of $r_{\mathrm{e}}, r_{\mathrm{d}}(\operatorname{arcsec}), \mu_{0}, \mu_{\mathrm{e}}(\mathrm{mag})$ and the RMS deviation to each fit. Error bars were calculated directly from the RMS scatter in luminosity along the fitted ellipse. f): Zoom of the central part of d), showing the fit and data set behaviour 
$87\left(\approx 210^{5} M_{\odot}\right)$, with ages of $\approx 5,6$ and 8 Myr, respectively. The models indicate that the $\mathrm{H} \alpha$ intensity of $12 \mathrm{Myr}$ old ionizing clusters, more massive than $510^{4} M_{\odot}$, should be detectable in our observations (sensitivity $I_{\mathrm{H} \alpha} \gtrsim$ $10^{37} \mathrm{erg} \mathrm{s}^{-1}$ ), nevertheless we fail to. It might indicate that clusters as massive as those produce enough Supernovae and massive star winds to blow away the surrounding gas, transforming the HII region after $9 \mathrm{Myr}$ in an optically thin one, which would produce an additional fadding of the emitting gas due to the leakage of ionizing photons. Regions 3 and 54 are just two border cases of the fadding process.

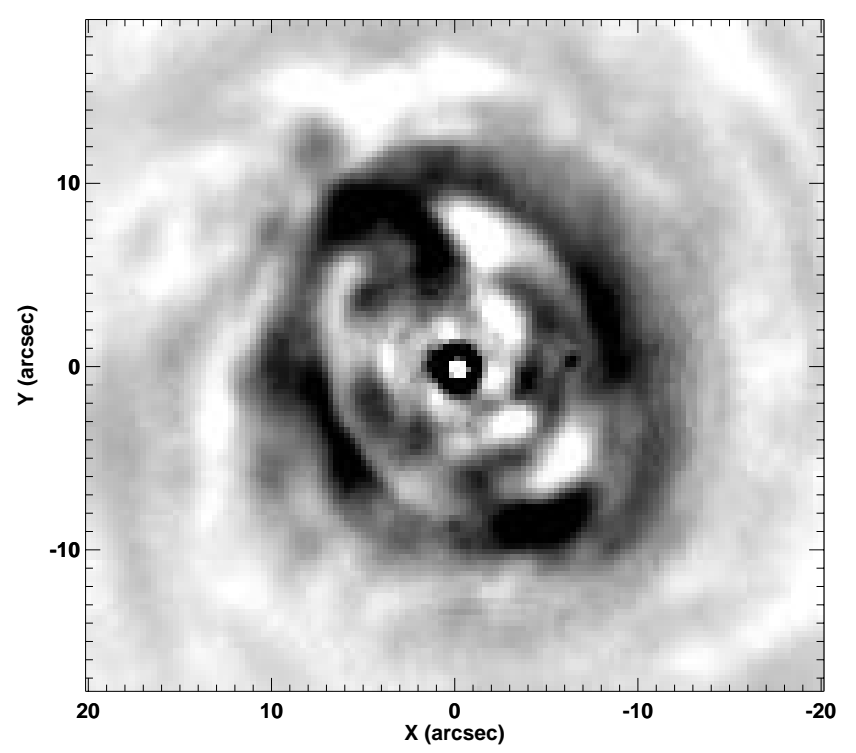

Fig. 15. Result of subtraction of a bulge +2 disks two dimensional model image from the $\lambda 6262$ image

\section{Surface brightness distribution}

In this section we study the monochromatic surface brightness distribution at $\lambda 3808 \AA, \lambda 4551 \AA, \lambda 5270 \AA, \lambda 6262 \AA$ and $\lambda 9183 \AA$ (see Table 1$)$, whose images are shown in Fig. 11. After masking disturbing field stars, ellipses were fitted to the isophotes following the algorithm described in Jedrzejewski (1987) and implemented in the ELLIPSE task of the IRAF.STSDAS package. The results for $\lambda 6262 \AA$ image is shown in Fig. 12. In Fig. 13 we plot the ellipticities and position angles (PA) of the fitted ellipses against its semi-major axis for the $\lambda 6262 \AA$ image. It presents the same trends already noted by Varela et al. (1990) for $B, V, R, I$ and $Z$ images and by MMG for near-infrared images. Also in our case the other continua images show essentially the same behaviour as in $\lambda 6262 \AA$. At $r \approx 10^{\prime \prime}$, where $\mathrm{PA} \approx 28^{\circ}$, the ellipticity has its first maximum $(\epsilon=0.21)$, suggesting an inner bar-like structure. It follows a transition zone at $10^{\prime \prime} \lesssim r \lesssim 25^{\prime \prime}$, where the ellipses are nearly round $(\epsilon \approx 0)$. Then, $\epsilon$ increases again, accompanied by a strong variation in PA from $25^{\prime \prime}$ to $35^{\prime \prime}$. With increasing radius, the ellipticity increases from 0.12 to 0.18 at $r \approx 60^{\prime \prime}$, the edge of our frames. MMG have found that $\epsilon$ increases further to 0.25 at $r \approx 120^{\prime \prime}$ and to $\sim 0.4$ at larger radii. The local maximum in the ellipticity at $50^{\prime \prime}$ corresponds to the external border of the inner ring, at $45^{\prime \prime}$. As mentioned by Kormendy (1993), these nested elliptical regions of different $\epsilon$ indicate that at most one of these zones can be axially symmetric and part of the disk must be oval, since warps can be excluded at this high surface brightness. Assuming axial symmetry for the zone between $r=50^{\prime \prime}$ and $70^{\prime \prime}$, we get an inclination angle of $i=35^{\circ}$. This behaviour of $\epsilon$ and PA is what one might expect of an oval or bar-like structure, more marked in young stars than in old populations, and with dust obscuration near the young component (Varela et al. 1990).

\subsection{Radial brightness profiles}

The mean surface brightness of the fitted ellipses are plotted against the ellipses major axis in Fig. 14. A least square test, using a downhill simplex minimization algorithm ("amoeba") implemented in the task NFIT1D of the IRAF package, shows that the best fit to these profiles are attained with a de Vaucouleurs' law, representing a small bulge, plus two Freeman's (1970) disks, a thick and a thin one. The de Vaucouleurs' law is given by

$\mu_{\mathrm{b}}(r)=\mu_{\mathrm{e}}+8.333\left[\left(\frac{r}{r_{\mathrm{e}}}\right)^{1 / 4}-1\right]$,

where $r_{\mathrm{e}}$ is the effective radius (the radius of the isophote that contains a half of the total bulge luminosity) and $I_{\mathrm{e}}$ is the surface brightness at $r=r_{\mathrm{e}}$. The Freeman's exponential disk law is

$\mu_{\mathrm{d}}(r)=\mu_{0}+2.5 \frac{r}{r_{\mathrm{d}}} \log I_{0}$,

where $r_{\mathrm{d}}$ is the disk scale length and $I_{0}$ is the luminosity density at $r=0$.

We express $\mu_{\mathrm{b}}$ and $\mu_{\mathrm{d}}$ in Oke \& Gunn's (1983) magnitude scale, where the parameters $\mu_{\mathrm{e}}$ and $\mu_{0}$ are given by

$$
\begin{aligned}
& \mu_{\mathrm{e}}=-2.5 \cdot \log I_{\mathrm{e}}-2.5 \cdot \log \frac{\lambda^{2}}{c}-48.60 \\
& \mu_{0}=-2.5 \cdot \log I_{0}-2.5 \cdot \log \frac{\lambda^{2}}{c}-48.60 .
\end{aligned}
$$

The respective parameters of each component are also given in Fig. 14. Sanders \& Tubbs (1980) also required the superposition of two disks plus a bulge, to explain the gas kinematics in NGC 5383 galaxy. 

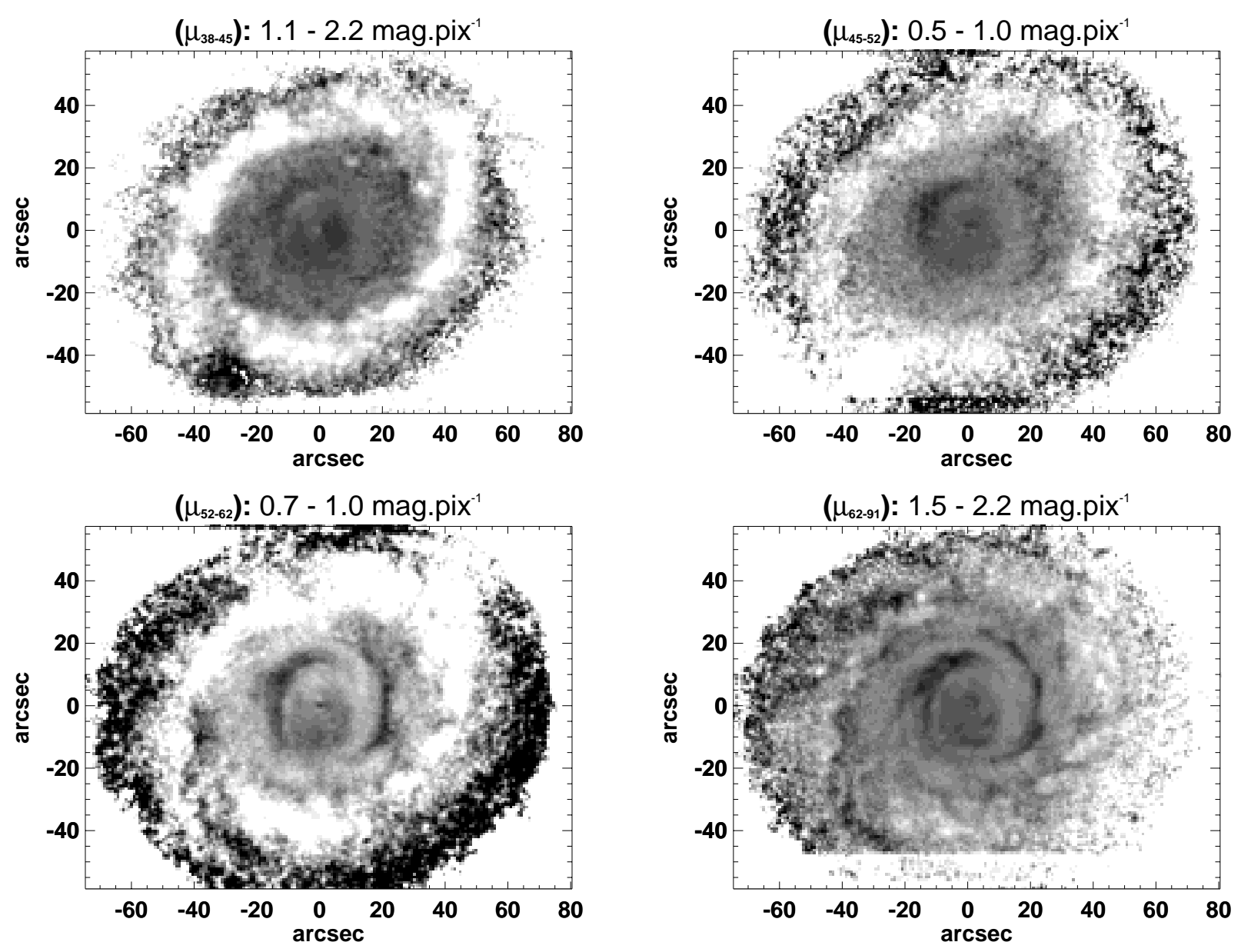

Fig. 16. Gray scale representation of two dimensional color maps $\mu_{38-45}, \mu_{45-52}, \mu_{52-62}$ and $\mu_{62-91}$. The limits are indicated in each image, white represents bluer colors. $(1$ pixel $=0.303$ arcsec $)$

\subsection{Rings in the central region}

The young stellar population underlying to the ring of HiI regions produces a bump in the observed continua profiles, which is more prominent in the $\lambda 3808 \AA$ profile (Fig. 14), where it extends itself between $30^{\prime \prime} \leq r \leq 55^{\prime \prime}$. In order to search for other substructures in the central regions, we generated a synthetic image from the profile fitted to $\lambda 6262 \AA$ image, addopting for each brightness level the ellipse shown in Fig. 12. This procedure is performed with the IRAF task BMODEL. The result of the subtraction of the model from the data for the central $20^{\prime \prime}$ is shown in Fig. 15. An oval ring of $20^{\prime \prime} \times 15^{\prime \prime}$ is detected, elongated in the same direction as the small central bar studied by MMG. It coincides with the isophote of highest ellipticity (Fig. 13) and settles the beginning of the strong variation in the isophotes position angle. At $r \sim 6^{\prime \prime}$ a smaller circular ring is found (Fig. 15), which coincides with the innermost ILR as calculated by Shaw et al. (1993). The central features inside $r<2^{\prime \prime}$ are artifacts of images subtraction.

\subsection{Two-dimensional color indexes}

We present in Fig. 16 the two-dimensional distribution of the color indexes $\mu_{38-45}=(\lambda 3808-\lambda 4551), \mu_{45-52}=$ $(\lambda 4551-\lambda 5270), \mu_{52-62}=(\lambda 5270-\lambda 6262)$ and $\mu_{62-91}=$ $(\lambda 6262-\lambda 9183)$. In these pictures, young (blue) and disk (red) populations appear as white and grey colors respectively. The population of the HII regions ring detaches clearly as white, in color indexes $\mu_{38-45}$ and $\mu_{52-62}$, becomes blured in $\mu_{45-52}$ and disappears in $\mu_{62-91}$. Inside the ring appear small arcs of dust, which present spirallike structure. A comparison with Fig. 1 reveals that HiI regions 55, 56 and 72 are located in the outer part of these dusty features to the northwest, probably indicating star formation activity induced by a spiral perturbation in the inner disk.

\subsection{Stellar population across the disk}

We obtained the continuum brightness distribution for five positions across the disk by integrating azimuthal profiles. These results are compared in Fig. 17 to combinations of Bica's (1988) templates, through a simple stellar 


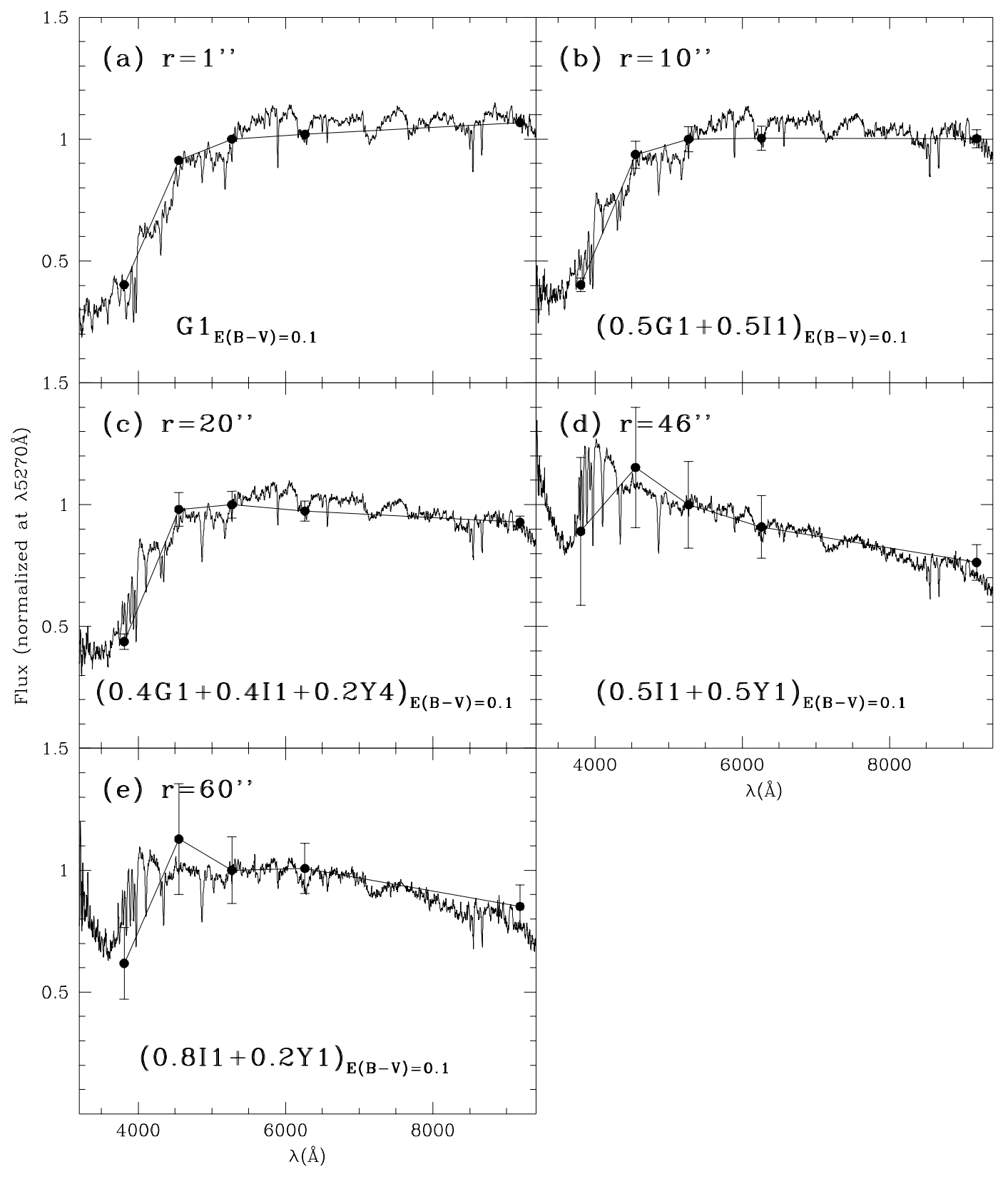

Fig. 17. Comparison of the continuum distribution with combinations of Bica's templates. We quote the contribution of each age template at $5270 \AA$. Bica's templates include star cluster spectra with different age and metallicity. Globular clusters: $G 1\left(\left[Z / Z_{\odot}\right]>0\right) ; G 2\left(\left[Z / Z_{\odot}\right]=-0.4\right) ; G 3\left(\left[Z / Z_{\odot}\right]=-1.0\right) ; G 4\left(\left[Z / Z_{\odot}\right]=-1.5\right) ; G 5\left(\left[Z / Z_{\odot}\right]=-1.9\right)$. Intermediate age: $I 1\left(10^{9}\right.$ yr); $I 2\left(510^{9} \mathrm{yr}\right)$. Young clusters: $Y 1\left(10^{7} \mathrm{yr}\right) ; Y 2\left(510^{7} \mathrm{yr}\right) ; Y 3\left(10^{8} \mathrm{yr}\right) ; Y 4\left(510^{8} \mathrm{yr}\right)$

population synthesis. From the bulge outwards it is possible to see a small increase of younger stellar population contribution, which appears massively on the ring, at $46^{\prime \prime}$. In the outer disk, at $60^{\prime \prime}$, there is a clear presence of a younger population than in the inner disk.

At the very center $\left(r \leq 1^{\prime \prime}\right)$ the population is an old one (Bica's template G1, > $10 \mathrm{Gyr}$ ); at $r=10^{\prime \prime}$ we need to introduce intermediate age population of $10^{9}-510^{9} \mathrm{yr}$ (template I1) in a similar amount than the old one; at $r=20^{\prime \prime}$ we need to add an even younger stellar popula- tion of $500 \mathrm{Myr}$ and at $r=46^{\prime \prime}$, the ring of Hil regions, we need a substantial contribution of $10^{7}$ years old stellar population, which corresponds to the continuum of ionizing clusters. The spectrum of the disk outside the ring of Hil regions $\left(r=60^{\prime \prime}\right)$, shows the presence of younger stellar population than the inner disk, showing that the star formation activity took place not only on the ring but also in the external disk. 


\section{Conclusions}

We carried out spectrophotometrically calibrated imagery of NGC 4736, with narrow band interference filters in emission lines and continua with high $\mathrm{S} / \mathrm{N}$ ratio.

We present a catalogue of $90 \mathrm{HII}$ regions with positions, sizes, emission line intensities and adjacent continua fluxes of $[\mathrm{OII}]_{\lambda \lambda 3727+3729}, \mathrm{H} \beta,[\mathrm{OIII}]_{\lambda 5007}, \mathrm{H} \alpha,[\mathrm{SII}]_{\lambda \lambda 6716+6730}$, and $[\mathrm{SIII}]_{\lambda \lambda 9070}$ lines. They are mainly distributed in a ring of $3.2 \mathrm{kpc}$ in diameter.

There is no HII region comparable to $30 \mathrm{DOR}$ in this galaxy, since the mean $\mathrm{H} \alpha$ luminosity is $L_{\mathrm{H} \alpha}=4.4 \times$ $10^{38} \mathrm{erg} \mathrm{s}^{-1}$.

The HII regions size distribution presents a characteristic diameter $D_{0}=115 \mathrm{pc}$ and follows the relation $\log \left(L_{\mathrm{H} \alpha}\right) \propto D^{3}$.

The physical properties of the set of Hil regions are: $<12+\log (\mathrm{O} / \mathrm{H})>=9.05$; temperatures of ionizing sources are in the range $3.410^{4} \lesssim T_{\star} \lesssim 4.010^{4} \mathrm{~K}$; masses of the ionizing clusters are in the range $510^{3} \lesssim M_{\mathrm{T}} / M_{\odot} \lesssim$ $210^{5}$. HII regions ionizing associations in NGC 4736 can reach masses up to $110^{5} M_{\odot}$. There are indications that the most massive ionizing associations, produce enough supernovae and/or massive star winds, as to fade out the ionizing gas, transforming the HII regions in an optically thin one.

The radial surface brightness distribution of the galaxy is better fitted by the superposition of a de Vaucouleurs', a thin and a thick exponential disk laws. This configuration was also found in NGC 5383. It was put in evidence an oval ring of $20^{\prime \prime} \times 15^{\prime \prime}$, elongated in the same direction as the small central bar.

The monochromatic colors show that oustside the ring of HII regions, the disk presents a contribution of a younger stellar population than that in its inner part.

Acknowledgements. We thank Dr. E. Bica for making available his templates of stellar population and Dr. R. CidFernandes who provided the "ET" code. The Jacobus Kapteyn Telescope is operated on the island of La Palma by the Royal Greenwich Observatory in the Spanish Observatorio del Roque de Los Muchachos of the Instituto de Astrofísica de Canárias. This project was partially supported by the spanish Dirección General de Investigación Científica y Técnica, grant n PB940433. This work was also partially supported by the brazilian institutions CNPq and FINEP.

\section{References}

Baker J.G., Menzel D.H., 1938, ApJ 88, 52

Barth C.S., Cepa J., Vílchez J.M., Dottori H., 1994, AJ 108, 2069

Beckmann J.E., Varela A.V.M., Muñoz-Tuñon C., Vílchez
J.M., Cepa J., 1991, A\&A 245, 436

Bica E., 1988, A\&A 195, 76

Bosma A., van der Hulst J.M., Sullivan III W.T., 1977, A\&A 57, 373

Burbidge E.M., Burbidge G.R., 1962, ApJ 135, 366

Burnstein D., Heiles C., 1984, ApJ 54, 33

Buta R., The Structure and Dynamics of Ringed Galaxies, 1984, Ph. D. Thesis, University of Texas, Austin

Buta R., 1988, ApJS 66, 233

Cid-Fernandez R., Dottori H., Viegas S.M., Gruenwald R., 1992, MNRAS 255, 165

Copetti M.F.V., Pastoriza M.G., Dottori H.A., 1986, A\&A 156, 111 (CPD)

de Vaucouleurs G., de Vaucouleurs A., Corwin H.G., 1976, Second Reference Catalogue of Bright Galaxies. University of Texas Press (RC2)

Filippenko A.V., Sargent W.L.W., 1985, ApJS 57, 503

Freeman K.C., 1970, ApJ 160, 811

Geller M.J., Huchra J.P., 1983, ApJS 52, 61

Hodge P.W., 1983, AJ 88, 1323

Hodge P.W., 1987, PASP 99, 915

Hodge P.W., Kennicutt R.C., 1983, AJ 88-3, 296

Jedrzejewski R.I., 1987, MNRAS 226, 747

Kennicutt R.C., 1988, ApJ 334, 144

Kennicutt R.C., Edgar B.K., Hodge P.W., 1989, ApJ 337, 761

Kormendy J., 1993, IAU Symposium 153, Galactic Bulges, Habing H., Dejonghe H. (eds.). Dordrecht, p. 209

Kurucz R.L., 1979, ApJS 40, 1

Lynds B.T., 1974, ApJS 28, 391

Maeder A., Meynet G., 1988, A\&AS 76, 411

McCall M.L., Rybski P.M., Shields G.A., 1985, ApJS 57, 1 (MRS)

Mendoza C., Zeippen C.J., 1982, MNRAS 198, 127

Möllenhoff C., Mathias M., Gerhard O.E., 1995, A\&A 301, 359 (MMG)

Mulder P.S., van Driel W., 1993, A\&A 272, 63

Oey M.S., Kennicutt R.C., 1993, ApJ 411, 137

Oke J.B., Gunn J.E., 1983, ApJ 266, 713

Osterbrock D.E., 1989, Astrophysics of Gaseous Nebulae and Active Galactic Nuclei. Univerity Science Books, Mill Valley, California

Pagel B.E.G., Edmunds M.G., Blackwell D.E., Chun M.S., Smith G., 1979, MNRAS 189, 95

Sandage A.R., Tamman G.A., 1974, ApJ 190, 525

Sanders R.H., Tubbs A.D., 1980, ApJ 235, 803

Shaw M.A., Combes F., Axon D.J., Wright G.S., 1993, A\&A 273,31

van den Bergh S., 1981, AJ 86, 1464

van der Kruit P.C., 1974, ApJ 188, 3

van der Kruit P.C., 1976, A\&A 52, 85

Varela A.M., Cepa J., Beckman J.E., Muñoz-Tuñon C., Vilchez J.M., 1990, Ap. Spa. Sci. 170, 305

Vilchez J.M., Pagel B.E.G., 1988, MNRAS 231, 257

Zaritsky D., Kennicutt R.C., Huchra J.P., 1994, ApJ 420, 87 